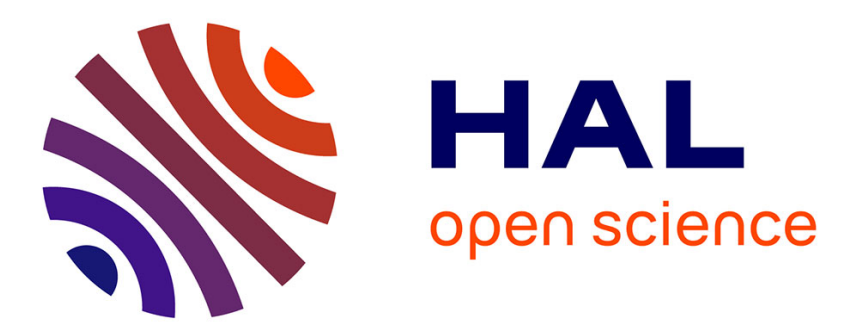

\title{
Modélisation des phénomènes électromagnétiques et mécaniques couples: application à l'analyse vibratoire des machines électriques
}

\author{
J.F. Imhoff, G. Reyne, A. Foggia, J.C. Sabonnadière
}

\section{To cite this version:}

J.F. Imhoff, G. Reyne, A. Foggia, J.C. Sabonnadière. Modélisation des phénomènes électromagnétiques et mécaniques couples: application à l'analyse vibratoire des machines électriques. Revue de Physique Appliquée, 1990, 25 (7), pp.627-648. 10.1051/rphysap:01990002507062700 . jpa-00246228

HAL Id: jpa-00246228

https://hal.science/jpa-00246228

Submitted on 1 Jan 1990

HAL is a multi-disciplinary open access archive for the deposit and dissemination of scientific research documents, whether they are published or not. The documents may come from teaching and research institutions in France or abroad, or from public or private research centers.
L'archive ouverte pluridisciplinaire HAL, est destinée au dépôt et à la diffusion de documents scientifiques de niveau recherche, publiés ou non, émanant des établissements d'enseignement et de recherche français ou étrangers, des laboratoires publics ou privés. 
Classification

Physics Abstracts

89.29

\title{
Modélisation des phénomènes électromagnétiques et mécaniques couples : application à l'analyse vibratoire des machines électriques
}

\author{
J. F. Imhoff, G. Reyne, A. Foggia et J. C. Sabonnadière \\ Laboratoire d'électrotechnique de Grenoble, U.R.A. C.N.R.S. 355, ENSIEG, BP 46, 38402 St-Martin- \\ d'Hères Cedex, France
}

(Reçu le 4 décembre 1989, accepté le 15 février 1990)

\begin{abstract}
Résumé. - La réduction des bruits et vibrations dans l'appareillage et les machines électriques préoccupe un grand nombre de constructeurs; la demande dans ce domaine se fait de plus en plus pressante. A travers nos travaux qui portent essentiellement sur les machines tournantes à courant continu, nous présentons une analyse qualitative, puis quantitative, des vibrations d'origine électromagnétique. Cette dernière nous a conduit au développement d'un logiciel de calcul de magnéto-mécanique basé sur la méthode des éléments finis, afin de permettre la prédiction des différents niveaux vibratoires en régime de fonctionnement permanent. Quelques exemples permettent d'illustrer la faisabilité du logiciel et montrent que les résultats obtenus sont très prometteurs.
\end{abstract}

\begin{abstract}
Manufacturers in electrical machinery are becoming highly interested in the reduction of noise and vibrations; this is due to the increasing awareness of the problem. In this paper, a qualitative and quantitative analysis of electromagnetic vibrations in D.C. machines is proposed. Then, the software which has been developed for the calculation of vibration levels in the case of steady state is presented and examples show that results are very promising.
\end{abstract}

\section{Introduction.}

Les vibrations et bruits engendrés par l'appareillage électrique et, en particulier, par les machines tournantes, font l'objet de nombreuses recherches. En effet, les moteurs, transformateurs, convertisseurs statiques et autres appareillages électriques sont devenus omniprésents. Les nuisances qui en résultent ont entraîné une exigence accrue des consommateurs et sensibilisé les industriels. Des contraintes spécifiques, comme la nécessaire "discrétion" des matérie $\mathrm{s}$ em arques, me en accen sur aspec vi ratoire qui est à la source de l'ensemble des nuisances constatées.

En particulier, le problème de la réduction voire de l'élimination des vibrations d'origine électromagnétique se pose avec une acuité certaine. Les nombreuses techniques de réduction (souvent empiriques) semblent avoir atteint leurs limites. Il apparaît nécessaire de chercher à les réduire à la source. Pour cela, une approche par la modélisation numérique est très intéressante. Elle implique une bonne compréhension des phénomènes, afin de pouvoir modéliser, aussi correctement que possible, les comportements électromagnétiques et mécaniques d'une structure.

Dans un premier temps, nous présentons le logiciel FLUXMECA qui permet ces différentes approches, ainsi que ses spécifications principales.

Puis, nous nous intéressons aux phénomènes à la source des vibrations électromagnétiques, c'est-àdire les forces électromagnétiques et en particulier celles, essentielles pour l'appareillage et les machines ournan es, qui s exercen su 'riau gnétiques.

Cette seule démarche permet d'étudier divers phénomènes comme par exemple l'influence de l'effet de pointe sur les forces électromagnétiques.

Cependant, l'étude, comme d'ailleurs la mesure, de grandeurs vibratoires et acoustiques nécessite pour être appréhendée correctement un traitement fréquence à fréquence grâce à une décomposition spectrale.

C'est la raison pour laquelle une approche rapide et qualitative est, dans un premier temps, proposée. 
Elle permet d'obtenir, pour des temps de calcul très raisonnables, une image des fréquences excitatrices ainsi que de leur amplitude. La raie d'encoche et ses harmoniques sont ainsi mis en évidence pour diverses structures tournantes.

Dans un second temps, l'étude est approfondie afin de répondre quantitativement aux attentes des utilisateurs. Cela implique la prise en compte de la rotation de la machine. Diverses solutions sont considérées et une technique est retenue. La résolution fréquence à fréquence des excitations déphasées qui en résultent permet d'obtenir le spectre électromagnétique de la machine étudiée.

Cette étude, assez complète, étayée de nombreux exemples d'application, permet de mettre en évidence l'énorme potentiel que la modélisation met au service des concepteurs de machines électriques. Mieux comprendre et quantifier les vibrations des structures est aujourd'hui devenu à portée de main.

\section{Caractéristiques générales du logiciel.}

Le logiciel de calculs d'électromagnétisme FLUXLAB développé au Laboratoire d'Electrotechnique de Grenoble (LEG) constitue la base du programme FLUXMECA. Comme le montre la figure 1, ce dernier est l'extension au domaine mécanique du premier qui permet d'effectuer l'ensemble des calculs magnétiques.

Ces logiciels reposent sur la méthode des éléments finis, tout à fait adaptée pour la résolution des problèmes électromagnétiques ou mécaniques. Le maillage qui y conduit est généré de manière entièrement automatique, suivant l'algorithme de Delaunay. Sur chaque élément fini, les fonctions d'interpolation sont $d u$ premier ou du deuxième ordre. Ces logiciels permettent d'effectuer des résolutions en magnéto-

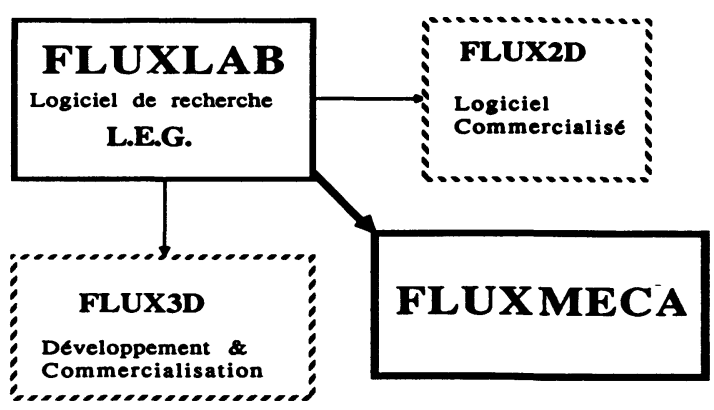

Fig. 1. - FLUXMECA et son contexte.

[FLUXMECA and its context.]

statique, en magnétodynamique dans le cas où il est nécessaire de prendre en compte les effets des courants induits, en mécanique statique et enfin en mécanique dynamique. Le logiciel FLUXMECA, bien que déjà très complet, a exigé des adaptations importantes pour permettre l'étude des vibrations électromagnétiques dans les machines tournantes.

COUPLAGE MAGNÉTO-MÉCANIQUE. - Le couplage réalisé dans FLUXMECA est uni-directionnel. La solution adoptée est un traitement successif des deux problèmes, magnétique puis mécanique; il s'agit en fait d'un simple transfert de données de l'électromagnétisme vers la mécanique.

En effet, les vibrations, en dehors des résonances, sont des déformations suffisamment faibles pour que les déplacements physiques soient négligeables quant à leur influence sur les grandeurs magnétiques. Cette constatation nous permet aussi de traiter les matériaux comme mécaniquement linéaires.

Analyse modale. - Cette partie est purement mécanique et consiste à déterminer les modes et fréquen-

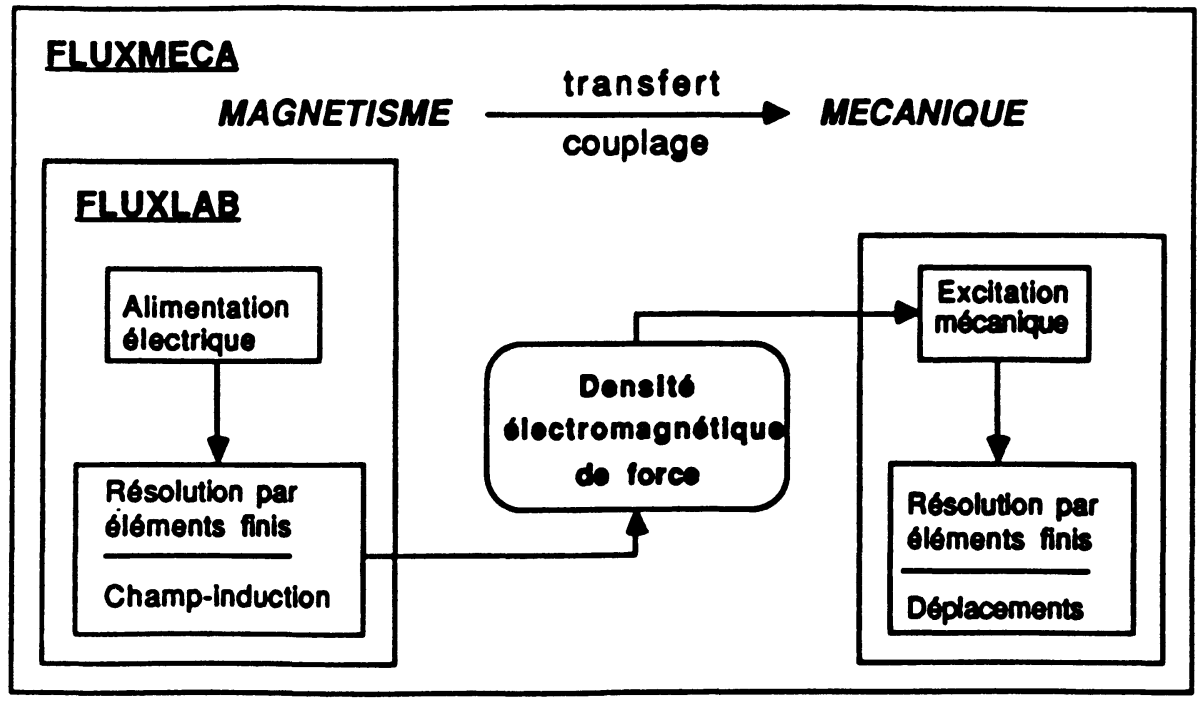

Fig. 2. - Synoptique de FLUXMECA.

[Diagramm of FLUXMECA.] 
ces propres d'une structure. Ces fréquences déterminées, il s'agira de les éviter par modification soit de la structure, soit des fréquences excitatrices. Rappelons que la connaissance des fréquences de résonance d'une structure est une donnée particulièrement importante quant à la conception d'une machine.

Couplage magnéto-mécanique. - L'autre partie du logiciel est le couplage lui-même, c'est-à-dire le calcul de l'excitation mécanique d'une structure à partir des grandeurs d'origine magnétique. Ce calcul est aisé pour les parties conductrices en cuivre, mais ne l'est pas pour les matériaux ferromagnétiques. Nous justifions dans la deuxième partie les raisons de cette difficulté, puis expliquons la manière dont nous l'avons résolue.

EQuATIONS. - Les équations de base utilisées dans FLUXMECA sont les suivantes :

- les équations magnétiques de Maxwell dans l'approximation du régime quasi-stationnaire.

$$
\begin{array}{rlrl}
\operatorname{D} \backslash(\mathbf{J}) & =0 & & \operatorname{Rot} \mathbf{E}+\partial \mathbf{B} / \partial t=0 \\
\operatorname{Div}(\mathbf{B}) & =0 & & \operatorname{Rot}(\mathbf{H})=\mathbf{J} \\
\mathbf{B} & =\boldsymbol{\mu} \mathbf{H}=\boldsymbol{\mu}_{0}(\mathbf{H}+\mathbf{M})
\end{array}
$$

$\mathbf{B}=$ induction magnétique

$\mathbf{M}=$ aimantation

$\mathbf{H}=$ excitation magnétique,

$\boldsymbol{\mu}=$ perméabilité magnétique,

$\mathbf{E}=$ champ électrique,

$\mathbf{J}=$ densité de courant .

- les équations de la mécanique des solides.

$$
\begin{aligned}
& \varepsilon_{1 \mu}=1 / 2 \cdot\left(\partial U_{i} / \partial x_{j}+\partial U_{i} / \partial x_{i}\right) \\
& \nu_{i,}=\left((1+\nu) \sigma_{i j}-\nu \sigma_{z z} \delta_{i j}\right) / E
\end{aligned}
$$

$\sigma_{i j, i}+f_{j}=0 \quad \sigma_{z z}=\operatorname{Tr}\left(\sigma_{i j}\right) \quad \sigma_{i j, i}=\Sigma \partial \sigma_{i j} / \partial x_{i}$

$\sigma_{i j}=$ contraintes,

$\varepsilon_{i j}=$ déformations,

$U_{i}=$ déplacements,

$f_{j}=$ forces volumiques,

$E=$ Module d'Young,

$\nu=$ coefficient de Poisson .

Principe de la résolution mécanique. - Les équations sont résolues par la méthode des éléments finis. Pour cela, le logiciel FLUXMECA applique le théorème de l'énergie potentielle totale $V$. Une analyse de Ritz avec le choix des déplacements aux nœuds comme coordonnées généralisées permet, pour une résolution statique, de ramener le problème de minimisation de la fonctionnelle à celui de la résolution d'un problème linéaire :

$$
\mathbf{K} \cdot \mathbf{Q}=\mathbf{F}
$$

soit sur chaque élément fini «e $»: \mathbf{K}^{\mathrm{e}} \cdot \mathbf{Q}^{\mathrm{e}}=\mathbf{F}^{\mathrm{e}}$

$$
\begin{aligned}
& \mathbf{K}=\text { rigidité de la structure }, \\
& \mathbf{Q}=\text { déplacements nodaux }, \\
& \mathbf{F}=\text { vecteur des forces équivalentes } .
\end{aligned}
$$

Le couplage magnéto-mécanique qui prend en compte les forces d'origine magnétique nécessite l'introduction, au sein du programme FLUXMECA, de la notion de surface. Il a fallu définir la surface d'un élément ferromagnétique, puis la force surfacique qui lui correspond. Cela s'est traduit au niveau de la résolution, par la prise en compte dans le système d'un nouveau terme dû à la contribution de ces forces surfaciques.

$$
F^{\mathrm{e}}=F_{\text {interne }}^{\mathrm{e}}+F_{\text {volume }}^{\mathrm{e}}+F_{\text {surface }}^{\mathrm{e}} .
$$

La résolution en régime dynamique, repose sur l'expression :

$$
M \mathbf{Q}^{\prime \prime}+C \mathbf{Q}^{\prime}+K \mathbf{Q}=\mathbf{F}(t)
$$

La prise en compte de l'amortissement n'est pas utile dans le cas des structures classiques utilisées en électrotechnique; le coefficient $C$ est, par conséquent, considéré comme nul. Le logiciel FLUXMECA se ramène alors à la résolution de :

$$
\mathbf{M Q}^{\prime \prime}+\mathbf{K Q}=\mathbf{F}(t) .
$$

Comme pour tout travail de ce type, nous procédons dans le domaine des fréquences à l'étude de chaque fréquence $f$ pour des grandeurs sinusoïdales. Ainsi, si nous utilisons les grandeurs complexes (italiques) à la fréquence $f$, nous obtenons :

$$
\left(-M \Omega^{2}+K\right) Q=F \quad \text { avec } \quad \Omega=2 \pi f .
$$

Cette formulation semble conduire à une résolution réelle, c'est-à-dire identique à celle correspondant au régime statique. Cependant les structures tournantes, en particulier, présentent une spécificité en regard de la plupart des autres types de structures mécaniques; les forces qui les excitent comprennent non seulement un grand nombre d'harmoniques, mais surtout, pour chacun d'eux, la répartition des forces se trouve déphasée dans le temps. Cela se comprend particulièrement bien pour un moteur où $x$ 'm le ar exem le sous un ôle alors qu'au même instant, à la même fréquence, elle sera presque nulle entre deux pôles. Cela conduit à résoudre l'équation : $\left(-M \Omega^{2}+K\right) Q=F$ où $Q$ et $F$ sont des grandeurs complexes.

A partir des informations qui découlent de la résolution, c'est-à-dire les déplacements aux nouds (vecteur $Q$ ), nous déduisons le déplacement en un point quelconque $P$, grâce à la matrice $[A]$ d'interpolation. La partie qui nous intéresse plus particulièrement est la prise en compte des forces de volume (dans les conducteurs par exemple) et de surface 
(sur les matériaux ferromagnétiques); pour cela, nous scindons les densités de forces en tout point en une somme de deux termes :

$$
\mathbf{F}=\mathbf{F}_{\mathrm{v}}+F_{\mathrm{s}}
$$

où $\mathbf{F}_{\mathrm{v}}$ et $\mathbf{F}_{\mathrm{s}}$ correspondent respectivement aux densités volumique et surfacique de force.

\section{Forces sur les matériaux ferromagnétiques.}

Le but de cette deuxième partie est de définir clairement le moyen d'obtenir les grandeurs mécaniques (forces puis contraintes) à partir des grandeurs magnétiques supposées connues: c'est le couplage magnéto-mécanique. Les matériaux ferromagnétiques captent et amplifient les phénomènes magnétiques et de ce fait, ils sont le siège des inductions les plus fortes; ils supportent donc l'essentiel des forces mécaniques et par conséquent, pour effectuer un calcul de vibrations aussi correct que possible, il est indispensable de connaître précisément la répartition de ces forces $[1,2]$.

Nous avons étudié les différentes méthodes qui permettent d'obtenir les forces et en avons déduit que seule l'approche énergétique conduit à une densité de force cohérente. Cette méthode prend en compte l'aspect thermodynamique et les grandeurs macroscopiques qui caractérisent le comportement global du matériau.

La base de la démonstration est l'application sur une déformation locale du principe des travaux virtuels. On peut en effet écrire que la force d'origine' magnétique est égale à l'opposé de la variation, à flux constant, de l'énergie magnétostatique globale par rapport aux déformations :

$$
\mathbf{F}=-\lfloor\partial W / \partial \xi\rfloor_{\varphi} .
$$

Ainsi, nous obtenons l'expression générale de la force :

$$
\begin{array}{r}
\mathbf{F}=\mathbf{J} \wedge \mathbf{B}-\Sigma\left(\alpha_{i} \nabla\left(\partial W / \partial \alpha_{i}\right)\right) \\
\mathbf{F}=\mathbf{J} \wedge \mathbf{B}-\nabla \Sigma\left[\alpha_{i}\left(\partial W / \partial \alpha_{i}\right)\right]+ \\
+\Sigma\left(\left(\partial W / \partial \alpha_{i}\right) \nabla \alpha_{i}\right)
\end{array}
$$

COMMENTAIRES. - Si la première expression est la plus lisible, c'est la seconde, plus explicite, qui a retenu notre attention. La force s'y décompose en 3 termes.

- Le premier $\mathbf{J} \wedge \mathbf{B}$ est la force de Laplace qui s'exerce sur toute distribution de courant.

- Le second $-\nabla \Sigma\left[\alpha_{i}\left(\partial W / \partial \alpha_{i}\right)\right]$ est l'opposé du gradient d'un scalaire qui exprime la dépendance de l'énergie vis-à-vis des différents paramètres physiques $\alpha_{i}$, caractéristiques du milieu; ce second terme est le gradient d'une pression scalaire.
- Le troisième terme, $\Sigma\left(\left(\partial W / \partial \alpha_{i}\right) \nabla \alpha_{i}\right)$ représente une force qui dépend des hétérogénéités de la matière, ou autrement dit des variations de ses paramètres physiques.

On peut montrer que la densité de force s'exprime sous la forme d'un gradient de tenseur:

$$
\mathbf{F}=\nabla(\underline{\tau})
$$

avec :

$$
\underline{\tau}=\underline{B} \underline{H}-\underline{I} \int(\mu-(\partial \mu / \partial \rho) \rho) H \mathrm{~d} H
$$

où $\mu$ est la perméabilité magnétique et $\rho$ la densité volumique de masse,

$$
\begin{array}{cc}
\underline{B} \underline{H}=\left[\begin{array}{lll}
B_{x} H_{x} & B_{y} H_{x} & B_{z} H_{x} \\
B_{x} H_{y} & B_{y} H_{y} & B_{z} H_{y} \\
B_{x} H_{z} & B_{y} H_{z} & B_{z} H_{z}
\end{array}\right] \\
\text { et } \underline{I}=\left[\begin{array}{lll}
1 & 0 & 0 \\
0 & 1 & 0 \\
0 & 0 & 1
\end{array}\right] .
\end{array}
$$

FORCES SURFACIQUES. - L'obtention de l'expression des forces d'origine magnétique qui s'exercent sur les surfaces des matériaux ferromagnétiques est d'autant plus importante que ce sont ces forces qui sont à l'origine de la majorité des contraintes.

\section{Expression.}

L'expression de la force surfacique $\mathbf{F}_{\mathrm{s}}$ est :

$$
\mathbf{F}_{\mathrm{s}}=\lim _{s \rightarrow 0}\left[\int \underline{\tau} \cdot \mathbf{n} \mathrm{d} s_{+}-\int \underline{\tau} \cdot \mathbf{n} \mathrm{d} s_{-}\right] / \mathrm{d} s .
$$

* Matériau linéaire, homogène, isotrope et incompressible. - Dans ce cas, nous pouvons démontrer que :

$$
\int(\mu-(\partial \mu / \partial \rho) \rho) H \mathrm{~d} H=\mathbf{H} \cdot \mathbf{B} / 2,
$$

d'où :

$$
\underline{\tau}=\left[\begin{array}{lll}
B_{x} H_{x}-\mathbf{H} \cdot \mathbf{B} / 2 & B_{y} H_{x} \\
B_{x} H_{y} & B_{y} H_{y}-\mathbf{H} \cdot \mathbf{B} / 2 \\
B_{x} H_{z} & B_{y} H_{z} \\
& \\
& B_{z} H_{x} \\
& B_{z} H_{y} \\
& B_{z} H_{z}-\mathbf{B} \cdot \mathbf{H} / 2
\end{array}\right] .
$$

Nous obtenons donc:

$$
\begin{aligned}
\mathbf{F}_{\mathrm{s}}=\mathbf{H}_{1}\left(\mathbf{B}_{1} \cdot \mathbf{n}\right)-\left(\mathbf{H}_{1} \cdot \mathbf{B}_{1} / 2\right) \mathbf{n}- \\
{\left[\mathbf{H}_{2}\left(\mathbf{B}_{2} \cdot \mathbf{n}\right)-\left(\mathbf{H}_{2} \cdot \mathbf{B}_{2} / 2\right) \mathbf{n}\right] . }
\end{aligned}
$$

A partir de cette forme très facilement exploitable, il est aisé de faire apparaître un résultat essentiel : la 
force est toujours normale à la surface du matériau considéré.

$\mathbf{F}_{\mathrm{s}}=\mathbf{H}_{1}\left(\mathbf{B}_{1} \cdot \mathbf{n}\right)-\mathbf{H}_{2}\left(\mathbf{B}_{2} \cdot \mathbf{n}\right)-$

$$
\left(\mathbf{B}_{1} \cdot \mathbf{H}_{1} / 2-\mathbf{B}_{2} \cdot \mathbf{H}_{2} / 2\right) \mathbf{n}
$$

$\mathbf{F}_{\mathrm{s}}=\left(\mathbf{H}_{1}-\mathbf{H}_{2}\right) \mathbf{B}_{\mathrm{n}}-\left(\mathbf{B}_{1} \mathbf{H}_{1} / 2-\mathbf{B}_{2} \mathbf{H}_{2} / 2\right) \mathbf{n}$

$\mathbf{F}_{\mathrm{s}}=\left(\mathbf{H}_{1 \mathrm{n}}-\mathbf{H}_{2 \mathrm{n}}\right) \mathbf{B}_{\mathrm{n}}-\left(\mathbf{B}_{1} \mathbf{H}_{1} / 2-\mathbf{B}_{2} \mathbf{H}_{2} / 2\right) \mathbf{n}$.

Une force d'attraction, dirigée vers l'air s'exerce sur la surface. Cela semble tout à fait logique, puisque l'énergie étant emmagasinée sous la forme $\mathbf{B} \cdot \mathbf{H} / 2$, la plus grande partie de l'énergie se trouve dans l'air et non dans le matériau.

*Matériau non linéaire et isotrope. - La non-linéarité magnétique est un phénomène fondamental en pratique quant à la détermination des propriétés des matériaux ferromagnétiques et de leur champ magnétique.

$$
\begin{array}{r}
\mathbf{F}=\nabla\left(\underline{B} \underline{H}-\underline{I} \int(\mu-(\partial \mu / \partial \rho) \rho) H \mathrm{~d} H\right) \\
\mathbf{F}=\nabla(\underline{B} \underline{H})-\Delta\left(\int \mu H \mathrm{~d} H\right)+ \\
\nabla\left(\int(\partial \mu / \partial \rho) \rho H \mathrm{~d} H\right) .
\end{array}
$$

Apparaissent clairement sur cette expression de la densité de force :

- l'expression $\nabla(\underline{B} \underline{H})$ qui peut s'écrire : $\nabla(\underline{B} \underline{H})=\mathbf{J} \wedge \mathbf{B}+\mu \nabla\left(\mathbf{H}^{2} / 2\right)$,

- le second terme : $\nabla\left(\int-\mu H \mathrm{~d} H\right)$ qui est le gradient de l'énergie magnétostatique,

- le troisième : $\nabla\left(\int(\partial \mu / \partial \rho) \rho H \mathrm{~d} H\right)$ qui compte pour l'influence de la magnétostriction.

Nous obtenons finalement :

$$
\begin{aligned}
\mathbf{F}_{\mathrm{s}}=\mathbf{H}_{1}\left(\mathbf{B}_{1} \cdot \mathbf{n}\right)-( & \left.\mu_{0} \mathbf{H}_{1}^{2} / 2\right) \mathbf{n}- \\
& \mathbf{H}_{2}\left(\mathbf{B}_{2} \cdot \mathbf{n}\right)-\quad \mu H \mathrm{~d} H \quad \mathbf{n}+ \\
& \left.\left(\int(\partial \mu / \partial \rho) \rho H \mathrm{~d} H\right)\right] .
\end{aligned}
$$

Ainsi, la prise en compte des propriétés réelles des matériaux conduit à l'introduction de termes nouveaux dans la force volumique, ce qui a pour effet de modifier l'expression des forces surfaciques.

Cependant l'ordre de grandeur physique (importance de la perméabilité magnétique relative) montre la prépondérance des contraintes liées aux forces surfaciques même pour un matériau légèrement saturé. Cette prépondérance est essentielle par la suite; en effet, elle nous permet d'affirmer que le comportement des matériaux est représenté avec une bonne précision par le terme principal de l'expression des forces surfaciques.

La validité de ces hypothèses a été confirmée par les données expérimentales obtenues lors d'une campagne de mesures sur un cadre d'Epstein. Une bonne corrélation a été obtenue entre les déformations mesurées de divers types d'échantillons (domaine magnétique linéaire et légèrement saturé) et les résultats correspondants obtenus par la modélisation.

EXEMPLE D'APPLICATION. - L'exemple qui suit est volontairement didactique afin de permettre de se familiariser avec les forces qui s'exercent sur les matériaux ferromagnétiques; il montre le type d'étude qui, à ce stade de nos travaux, peut déjà être mené. En l'occurrence, il s'agit de chiffrer l'importance de l'effet de pointe ou plutôt d'arête vive.

Etude de la densité surfacique de force au voisinage d'une arête vive. - Pour faire cette étude, nous avons choisi une dent simplifiée de rotor de machine, située en face d'un pôle inducteur statorique ou, plus exactement, une demi-dent pour une raison de symétrie ; l'arête vive de la demi-dent est en fait un arrondi (arc de cercle) dont nous avons fait varier le rayon de courbure, en ayant pris soin de maintenir le flux constant afin d'obtenir des résultats comparables.

Nous avons choisi un entrefer $e=1 \mathrm{~mm}$ et nous avons imposé un flux de manière à ce que l'induction $B_{e}$ dans l'entrefer soit de 1.3 Tesla au milieu de la dent, tout du moins pour des rayons de courbure $r$ faibles devant l'entrefer $e$.

La figure 3 montre la répartition de la densité surfacique de force sur la dent et, en particulier, au niveau de l'arête pour différentes valeurs de $r / e$.

Une étude succinte sur l'influence de l'arête vive d'une dent de rotor de machine tournante sur la densité surfacique de force permet de mettre en évidence que plus une arête est vive, plus la densité de force est importante dans son voisinage.

Localement, les déformations mécaniques qui en résultent sont plus importantes et pourtant, globalement, il est aci e e veri 1er que a orce tota e qu s'exerce sur la dent reste inchangée; les arêtes vives apparaissent comme défavorables sur le plan vibratoire même si leurs effets sont atténués par la saturation dans les matériaux magnétiques; il convient de les arrondir légèrement bien que leur influence sur le comportement vibratoire de la machine ne soit pas très important. Par ailleurs, il est intéressant de noter que les arêtes vives au niveau du rotor n'ont pas d'influence directe sur le pôle pour des machines avec des entrefers de dimensions usuelles. 

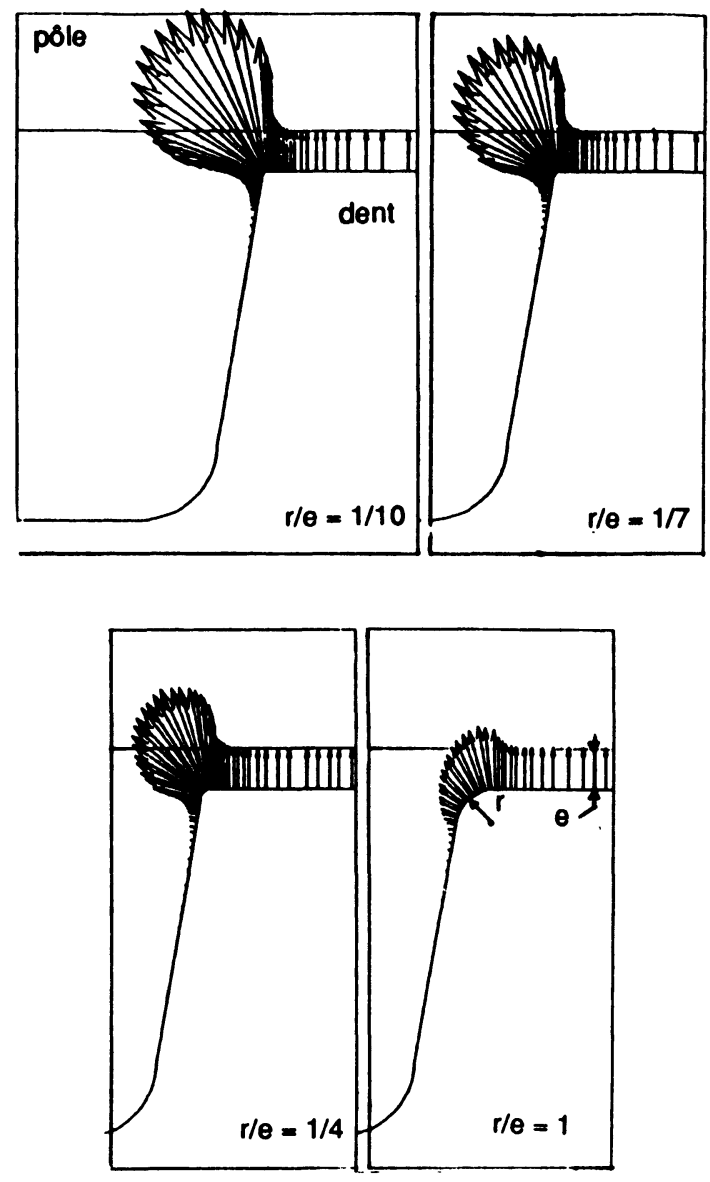

Fig. 3. - Répartition de la densité surfacique de force sur la dent.

[Distribution of the surface force density on a tooth.]

\section{Analyse de la répartition des forces.}

Diverses structures sont ici considérées. Bien qu'il s'agisse essentiellement de structures tournantes, l'étude est, dans cette partie, conduite en statique c'est-à-dire en l'absence du mouvement de parties mobiles. Nous verrons, dans la partie suivante, le traitement complet de cas de vibrations de structures avec prise en compte de la rotation et de la réponse propre de la structure. Dans l'immédiat, nous simplifions le problème en nous limitant à l'étude spatiale de la répartition des forces, principalement dans l'entrefer de la machine. L'analyse harmonique de cette répartition des forces est faite, l'influence de la saturation ou de divers paramètres géométriques chiffrée. De précieux renseignements peuvent être obtenus par ce biais en s'affranchissant de la nécessité de mener de lourds calculs.

EXEMPLE 1 : ETUDE DE L'EFFET D'ENCOCHE. - Les variations brutales de perméabilité magnétique dues à l'encochage du rotor entraînent une forte modulation de l'induction magnétique et par conséquent des forces au sein de toute machine tournante. Cet effet dit « effet d'encoche » se retrouve dans tous les types de machines électriques tournantes. Divers procédés sont couramment utilisés pour réduire l'importance des excitations électromagnétiques responsables de la « raie d'encoche » et de ses harmoniques. D'une part, on utilise l'inclinaison des encoches ou les " chevrons » et, d'autre part, on joue sur la forme des encoches en les fermant plus ou moins. Cependant, «l'effet d'encoche », d'origine électromagnétique, de par son importance, reste l'un des problèmes clef de la réduction des vibrations des machines électriques. Afin d'illustrer ce phénomène, nous mettons en évidence sur une géométrie simplifiée, l'influence de la largeur de l'entrefer, de la saturation puis de la semi-fermeture des encoches sur la répartition de la densité de force.

- Influence de l'entrefer. - Dans une machine tournante, l'entrefer, inévitable, "consomme " une part importante de l'énergie électrique fournie à la machine. Il faut donc un entrefer aussi faible que possible. Or plus l'entrefer est fin, plus la modulation ou l'ondulation de la densité de force est importante ; si $e$ est la largeur d'entrefer et $d$ la largeur de dent, les figures $4 a, 4 b$ et $4 c$, obtenues dans le cas d'une géométrie simplifiée pour différents rapports $e / d$, à ampères-tours constants, permettent d'illustrer ce phénomène

Une étude plus détaillée, dans le cas linéaire, permet de relier le rapport $(e / d)$ au rapport $\left(F_{\min } / F_{\max }\right)$ où $F_{\min }$ est la valeur minimale (au creux de l'ondulation) de la densité de force et $F_{\max }$ sa valeur maximale; à condition que le flux soit bien canalisé par les dents $(e / d<50 \%)$, nous avons la loi approximative : $F_{\min } / F_{\max } \cong e / d$. Ce rapport est d'autant plus petit que l'ondulation est plus forte.

- Influence de la saturation. - Par ailleurs, pour un entrefer donné, nous pouvons vérifier qu'un fonctionnement en régime légèrement saturé permet de réduire l'ondulation de la densité de force. Cela est particulièrement net au niveau de la corne polaire qui présente généralement une faible rigidité mécanique. Cet effet ne peut être que bénéfique sur le plan vibratoire. Par exemple, pour $e / d=20 \%$, la figure $5 a$ représente la répartition de la densité de force sur le pôle en régime saturé.

Après avoir calculé le flux utile par pôle en régime saturé, nous avons déduit les ampères-tours qui permettent d'obtenir, en régime linéaire, le même flux ; la figure $5 \mathrm{~b}$ montre la répartition de la densité de force correspondante. Sans qu'il soit nécessaire de comparer les déformées correspondantes, nous pouvons prévoir que le comportement vibratoire de la machine légèrement saturée sera meilleur que celui de la machine en régime linéaire.

- Influence de la fermeture des encoches. Plutôt que d'augmenter la largeur de l'entrefer d'une machine au prix de dégrader notoirement ses performances, il est préférable de se tourner vers une autre 


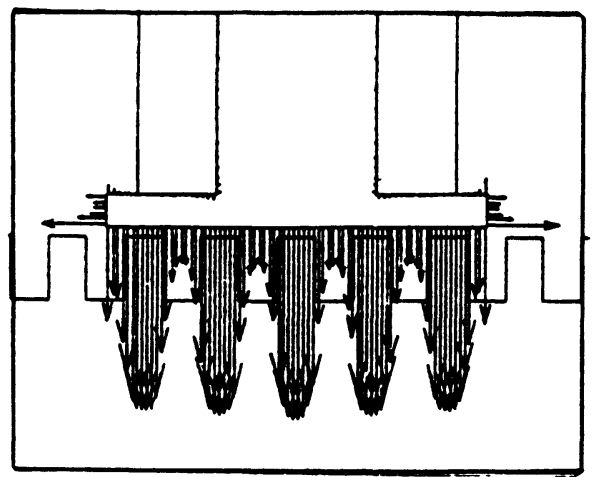

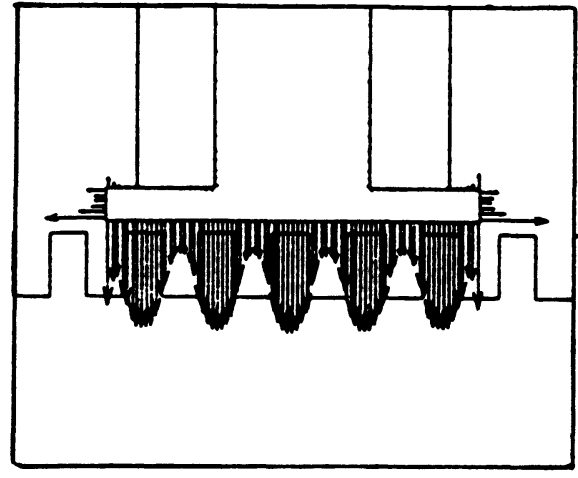

h)

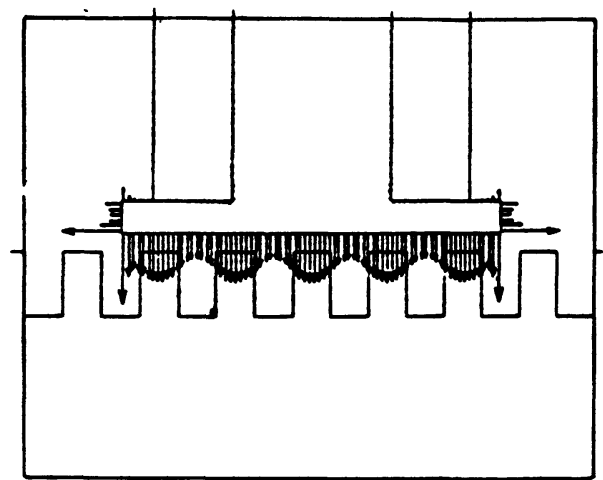

(')

Fig. 4. - (a) Densité de force pour $e / d=25 \%$. (b) Densité de force pour $c / d=30 \%$. (c) Densité de force pour $e / d=50 \%$.

[(a) Force density for $e / d=25 \%$. (b) Force density for $e / d=30 \%$. (c) Force density for $e / d=50 \%$.]

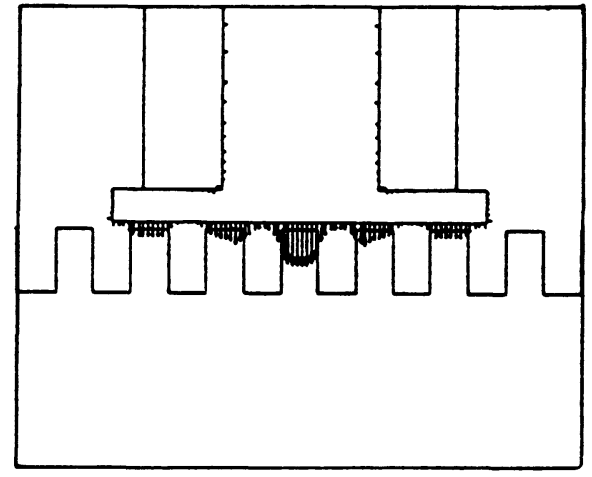

1)

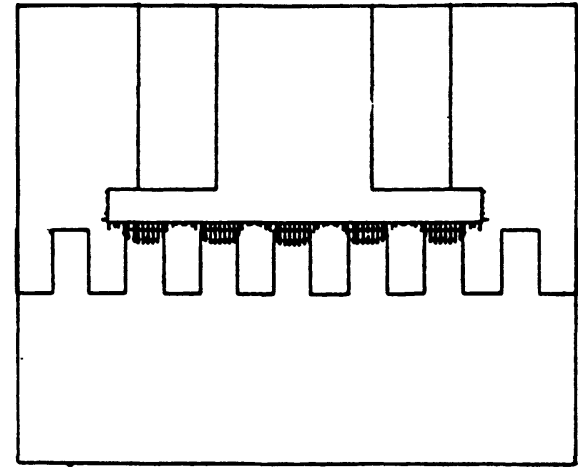

b)

Fig. 5. - (a) Régime saturé. (b) Régime linéaire.

[(a) Distribution of the force density with saturation. (b) Distribution of the force density in the linear case.]

solution qui consiste à fermer plus ou moins les encoches.

Les figures $6 a, 6 b$ et $6 c$, obtenues à ampères-tours constants, montrent très nettement que l'ondulation de la densité de force est d'autant plus faible que les encoches sont davantage fermées; en effet, le rapport $F_{\min } / F_{\max }$ vaut respectivement $24 \%, 56 \%$ et $78 \%$. Cette technique de fermeture des encoches ne donne de bons résultats sur le plan vibratoire que si la saturation des matériaux magnétiques n'est pas trop élevée; en effet, dans le cas contraire, les isthmes de fermeture se saturent fortement et deviennent inefficaces.

Le traitement des quelques aspects présentés cidessus montre la souplesse de ce type d'analyse ainsi que l'intérêt de pouvoir chiffrer rapidement 

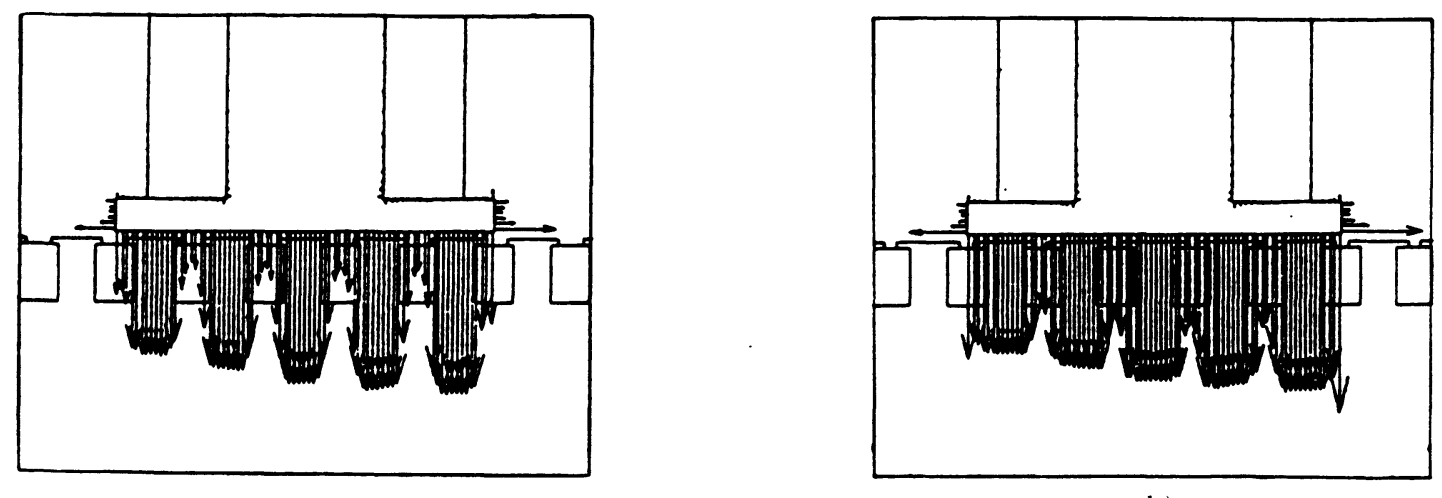

(1)

h)

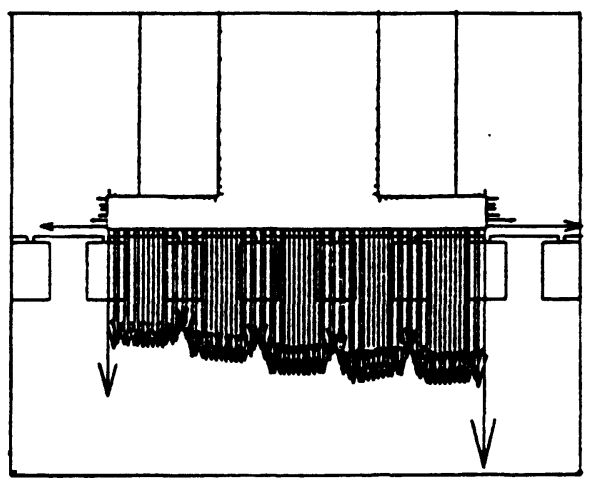

c)

Fig. 6. - (a) Encoches semi-fermées (30\%). (b) Encoches semi-fermées (60\%). (c) Encoches semi-fermées $(80 \%)$.

[(a) Half closed slots $(30 \%)$. (b) Half closed slots $(60 \%)$.

l'influence de divers paramètres. La multiplication des applications possibles impose, ici, une limitation des « cas » traités.

EXEMPLE 2 : RÉPARTITION DES FORCES DANS UNE MACHINE TOURNANTE. - L'analyse des harmoniques d'espace du carré du module de l'induction $\left(B^{2}\right)$ le long d'un cercle situé dans l'entrefer de la machine donne une bonne image des principales composantes vibratoires qui excitent directement soit les pôles du stator, soit les dents du rotor. En effet, compte tenu de ce que l'expression de la densité surfacique de force sur un matériau ferromagnétique est proportionnelle au carré de l'induction, la connaissance des spectres de $B^{2}$ permet de mettre en évidence l'importance de l'effet d'encoche ou de chiffrer l'influence d'un balourd sur le spectre des forces.

Structure simplifiée d'une machine à courant continu. - A titre d'exemple, nous présentons tout d'abord le cas d'une machine à courant continu simplifiée comportant quatre pôles et trente trois encoches au rotor. La figure 7a représente la répartition de la densité de force qui s'exerce sur le stator et, à côté, le spectre de $B^{2}$ correspondant.

Ce spectre purement électromagnétique fait apparaître plusieurs raies caractéristiques (numérotées 1, $2, \ldots)$.
- La raie $n^{\circ} 1$ (harmonique 4 de la fréquence de rotation) est liée à la présence des pôles et elle correspond au fondamental des efforts qui s'exercent sur le rotor. Cependant des mesures expérimentales semblent montrer que cette raie qui excite essentiellement le rotor ne fait pas vibrer le stator de manière significative. Ce ne serait pas le cas pour d'autres types de machines comme par exemple les machines synchrones.

- La raie $n^{\circ} 2$ (harmonique 33 de la fréquence de rotation) est due à l'encochage du rotor. Comme l'excitation se fait directement sur le pôle, l'ensemble de la structure est mis en vibration. C'est la « raie d'encoche », essentielle, dont apparaît aussi le premier harmonique. Il est à noter la présence de modulations de ces raies par la raie $n^{\circ} 1$.

- La raie de "balourd" de rang 1 (fréquence de rotation) est, aux erreurs numériques près, nulle ce qui traduit l'absence de balourd mécanique ou magnétique dans cet exemple.

Apparition d'un balourd. - Si nous introduisons un léger excentrement du rotor (vers le bas par exemple), cela entraîne une dissymétrie au niveau de la répartition de la densité de force (Fig. 8a) et l'apparition très nette de la raie de «balourd ». Le spectre de la figure $8 \mathrm{~b}$ est beaucoup plus dense que précédemment : les effets de modulation sont renforcés. Cela est logique puisque la raie de «balourd » vient 


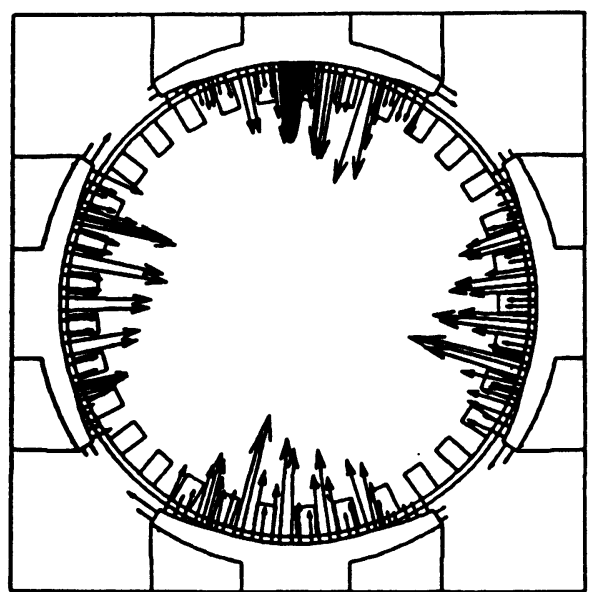

a)

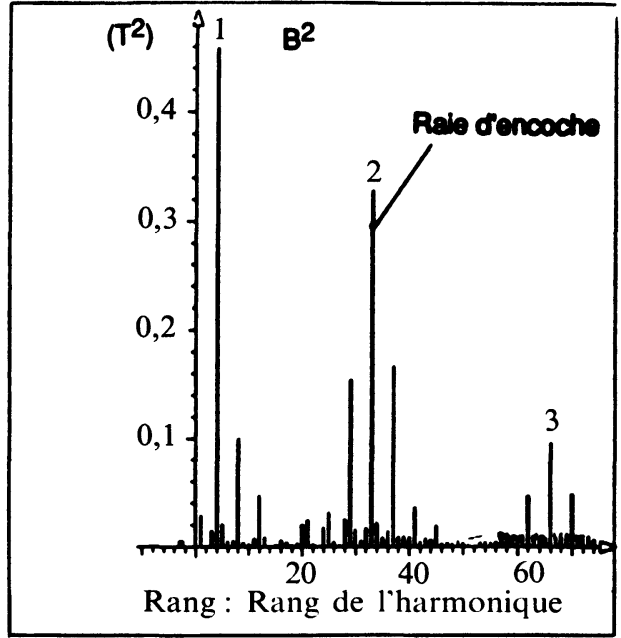

W)

Fig. 7. - (a) Densité surfacique de force. (b) Harmoniques d'espace de $B^{2}$.

[(a) Surface force density. (b) Spatial harmonic components of $B^{2}$.]

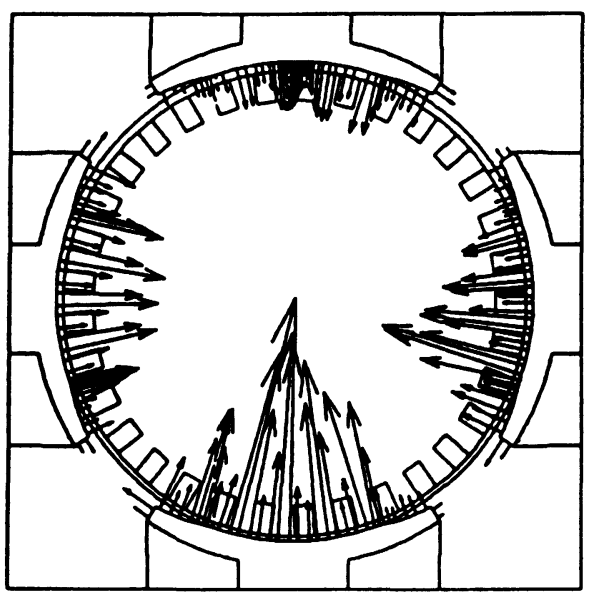

a)

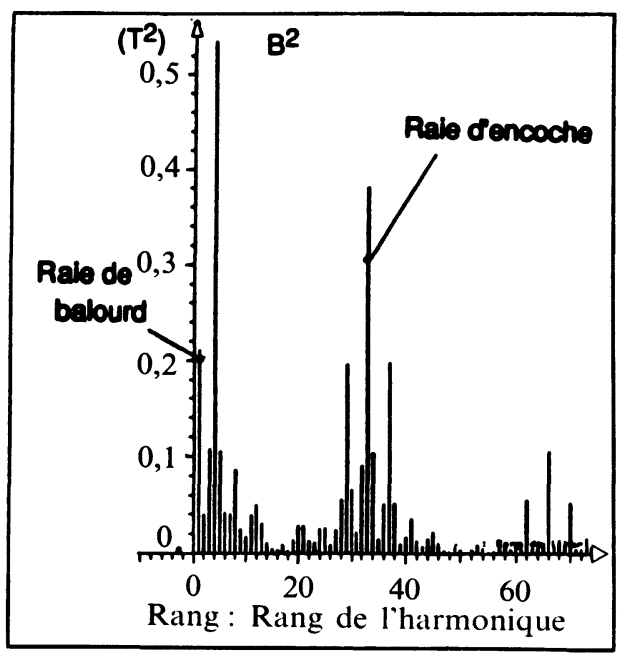

(1)

Fig. 8. - (a) Densité surfacique de force. (b) Harmoniques d'espace de $B^{2}$.

[(a) Surface force density. (b) Spatial harmonic components of $B^{2}$.]

maintenant moduler les raies dues aux encoches ou éventuellement aux ôles.

Plutôt qu'exciter la raie de «balourd" par une excentration mécanique du rotor, nous pouvons introduire plus simplement une dissymétrie magnétique ou électrique en réduisant par exemple les ampères-tours d'un bobinage inducteur sur un pôle ou en modifiant localement la perméabilité de certains matériaux. Les spectres de $B^{2}$ que l'on obtient alors ont sensiblement le même aspect que les précédents.

Par ailleurs, en faisant fonctionner la machine à courant continu en régime légèrement saturé, nous avons pu vérifier que la saturation des tôles magnéti- ques semble améliorer le comportement vibratoire. En effet, l'am litude de la raie d'encoche ar rapport à celle des pôles diminue. Cela signifie que, pour une force globale par pôle comparable, l'effet d'encoche sera atténué. Ce résultat confirme la tendance à l'amélioration en régime saturé déjà signalée au paragraphe précédent.

Machine à courant continu industrielle. - Une coupe de moteur industriel, donc plus réelle, est traitée de la même manière que précédemment. La forme des encoches est plus soignée, arrondie et les encoches sont semi-fermées comme le montre la figure $9 \mathrm{~b}$. A titre indicatif, la figure $9 \mathrm{a}$ représente la répartition de la densité de force sur les pôles. 


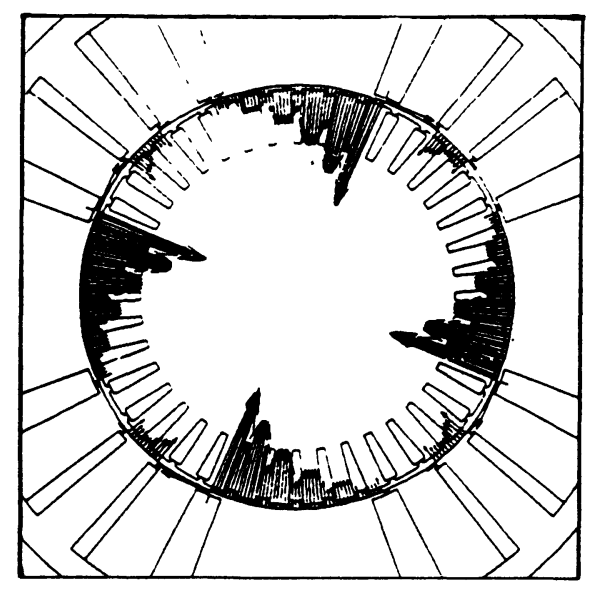

a)

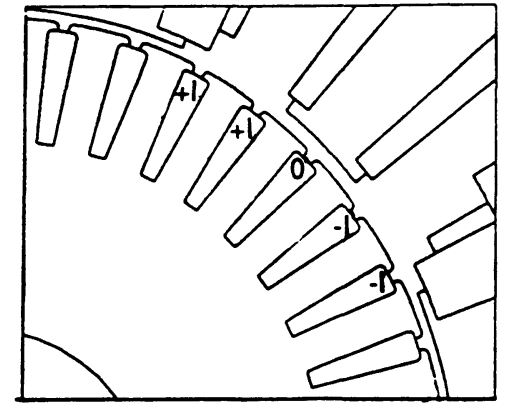

b)

Fig. 9. - (a) Coupe du moteur et répartition de la densité de force. (b) Détail du moteur.

[(a) Cross-section of the motor and distribution of the force density. (b) Slots of the motor.]

La raie d'encoche est très visible sur le spectre de la figure 10 et coïncide avec l'harmonique de rang 40 qui correspond au nombre d'encoches. Le rapport $\mathrm{H} 40 / \mathrm{H} 4$ de la raie d'encoche au fondamental des forces vaut environ $40 \%$. Dans le cas de l'autre moteur, il est facile de voir que ce rapport égal à $\mathrm{H} 33 / \mathrm{H} 4$ vaut $60 \%$. Les entrefers étant du même ordre de grandeur, cette différence s'explique essentiellement par le fait que le moteur industriel cidessus possède des encoches semi-fermées; ces dernières permettent d'atténuer la raie d'encoche de manière significative pourvu que les tôles magnétiques ne soient pas trop saturées.

Pour atténuer encore davantage la raie d'encoche, nous proposons de remplacer les conducteurs classiques du rotor par des conducteurs légèrement perméables ; l'effet observé est particulièrement efficace comme le montre la figure $11 \mathrm{~b}$ (cas du moteur simplifié).

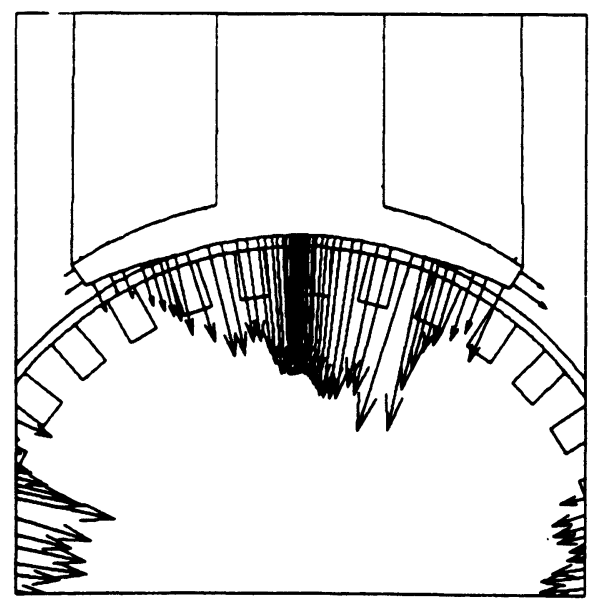

a)

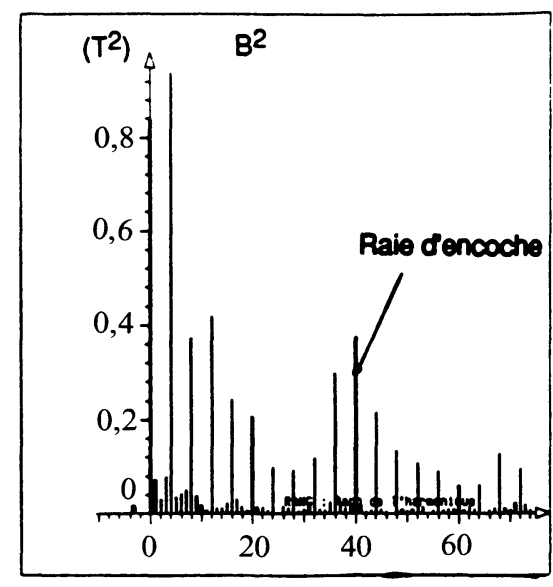

Fig. 10. - Spectre de $B^{2}$.

[Spectrum of $B^{2}$.]

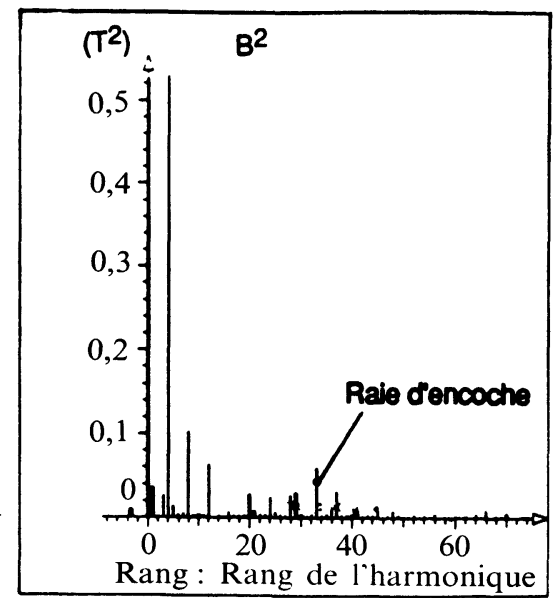

b)

Fig. 11. - (a) Densité de force avec des conducteurs perméables. (b) Spectre de $B^{2}$ correspondant.

[(a) Force density in the case of magnetic conductors. (b) Corresponding spectrum of $B^{2}$.] 
Ces résultats correspondent au cas où la perméabilité magnétique relative des conducteurs est de 10 ; il n'est pas utile qu'elle soit très élevée pour que les chocs magnétiques dus aux encoches soient très fortement atténués. De plus, cette technique contribue à augmenter légèrement le couple. Actuellement, de tels conducteurs ne sont pas commercialisés en France, mais il est à noter qu'ils font l'objet de recherches très approfondies au Japon.

\section{Fonction de transfert électromécanique en régime dynamique.}

L'analyse de la répartition des efforts permet l'étude de l'influence de certains paramètres et permet aussi de mieux comprendre les mécanismes de génération des vibrations. Cependant, elle n'est pas toujours suffisante. Ainsi, pour faire un calcul quantitatif de vibrations, il est indispensable de calculer la fonction de transfert électromécanique, c'est-à-dire la réponse mécanique de la structure aux exitations mécaniques [3, 4]. En effet à partir d'une analyse locale des forces, il est difficile de prévoir le comportement global de la machine; la rotation ou la réponse de la structure intervient. Certaines fréquences peuvent être complètement atténuées ou au contraire amplifiées, en particulier lorsqu'il y a des phénomènes de résonance.

Rappelons qu'un module de FUXMECA permet le calcul des fréquences propres de la structure. Le calcul ultérieur de la réponse mécanique de la structure à une excitation électromagnétique ne pourra se faire qu'en dehors de ces fréquences. En effet, pour celles-ci, l'amplitude des vibrations devient telle que notre modèle linéaire, sans prise en compte de l'amortissement et sans couplage complet (non interaction supposée des déformations mécaniques sur les valeurs des grandeurs électromagnétiques) ne permet plus de répondre. Cela d'ailleurs n'est pas gênant, car en pratique le fonctionnement d'une structure à la résonance est proscrite. Il suffit donc, expérimentalement comme en modélisation, de s'assurer que le fonctionnement n'excite pas de fréquence propre.

CALCUL DES VIBRATIONS ÉLECTROMAGNÉTIQUES. - Le principe de calcul de la fonction de transfert respecte le schéma de FLUXMECA présenté en première partie sur la figure 2 ; nous commençons par résoudre le problème magnétique qui permet de calculer l'évolution de la répartition de la densité de force électromagnétique dans le temps et ensuite nous effectuons le transfert de données, de manière transparente pour l'utilisateur, vers la mécanique pour déterminer les déplacements et donc les vibrations correspondantes.

Quel que soit le type d'alimentation, les excitations mécaniques, c'est-à-dire les forces électromagnétiques au sein de la machine évoluent dans le temps lorsque le rotor tourne. Un exemple de répartition des forces sur un pôle statorique à deux instants $t=0$ et $t=\Delta t$ de rotation proches est donné sur les figures $12 \mathrm{a}$ et $12 \mathrm{~b}$.

C'est pourquoi le calcul de la fonction de transfert a été décomposé en quatre étapes :

Première étape. - Permettre de prendre en compte le mouvement de parties mobiles, soit en l'occurrence d'un rotor de machine à courant continu. Cela impose que des pièces maillées puissent se déplacer l'une par rapport à l'autre sans que le maillage entre les deux ne soit, par ses déformations, source de difficultés.

Deuxième étape. - Calculer les différents états magnétiques de la machine, correspondant aux différentes positions du rotor. Nous en déduisons la répartition des forces pour chaque pas de temps. Cette succession de répartitions spatiales donne une série d'échantillons que nous appellerons « échantillons temporels".

Troisième étape. - Calculer, en chaque point, par une décomposition en série de Fourier, à partir des

Fig. 12. - (a) Densité de force à $t=0$. (b) Densité de force à $t=\Delta t$.

[(a) Force density at instant of time $t=0$. (b) Force density at instant of time $t=\Delta t$.] 
échantillons temporels, les harmoniques de force qui s'exercent sur la structure; la théorie de l'échantillonnage apporte une solution simple et rigoureuse. Ainsi, nous obtenons une autre série d'échantillons que nous appellerons "échantillons fréquentiels". Chacun d'entre eux représente, pour une fréquence donnée, la répartition spatiale des forces excitatrices ; ces forces sont, à la fréquence considérée, connues en amplitude et en phase.

Quatrième étape. - Effectuer, pour chaque échantillon fréquentiel, une résolution mécanique du problème. Cette résolution nous donne l'ensemble des grandeurs vibratoires à cette fréquence. $\mathrm{Si}$ le comportement vibratoire global veut être connu, il apparaît donc nécessaire d'effectuer autant de résolutions mécaniques qu'il y a d'échantillons fréquentiels. Le résultat peut être présenté sous forme de spectre'des vibrations pour chacun des points de la machine.

Les hypothèses qui nous permettent de procéder ainsi sont les suivantes :

- les courants induits sont négligés. En conséquence, les états magnétiques de la machine à chaque instant sont régis par les équations de la magnétostatique ;

- le régime de fonctionnement est permanent. Les excitations mécaniques sont périodiques dans le temps. Les différents pas de temps doivent couvrir une période temporelle pour les phénomènes considérés ;

- la décomposition en série de Fourier est possible car nous travaillons dans le domaine de la mécanique linéaire.

Le principe de calcul de la fonction de transfert apparaît sur la figure 13 .

DÉCOMPOSITION EN SÉRIE DE FOURIER. - Elle est d'autant plus intéressante qu'elle permet d'isoler de l'ensemble des vibrations la ou les fréquences qui nous intéressent plus particulièrement, par exemple, la raie d'encoche ou l'un de ses harmoniques. De plus, le nombre des composantes harmoniques dont il faut tenir compte n'est pas très élevé en général pour les machines classiques. En fonction des phénomènes étudiés, c'est le théorème de Shannon qui nous impose le nombre d'échantillons minimal à prendre en compte.

Les coefficients de Fourier peuvent donc être évalués par un calcul direct, dérivé de la transformée de Fourier discrète ou par une FFT (Transformée de Fourier rapide). Dans le cas où il est nécessaire de connaître l'ensemble des coefficients de Fourier, la FFT permet d'obtenir les résultats beaucoup plus rapidement que le calcul direct. Par contre, si nous nous intéressons à une fréquence particulière, le calcul direct peut être avantageux. Nous l'utilisons très largement pour nos calculs de vibrations dans FLUXMECA au niveau du module d'intégration éléments finis. Notons que pour utiliser la FFT classique (à base 2), il faut disposer d'un nombre d'échantillons égal à une puissance entière de 2 ; cela est assez contraignant au niveau du nombre et donc du temps de calcul.

PRISE EN COMPTE DE LA ROTATION. - Le problème est lié aux éléments finis et à leur déformation en cas de mouvement relatif de plusieurs pièces entre elles. Un exemple simple est donné sur les figures $14 a$ et $14 b$.

Les éléments finis qui se déforment sont situés dans l'entrefer. Ils deviennent très rapidement de mauvaise qualité, aplatis ou retournés. La résolution par la méthode des éléments finis devient impossible ou alors la précision des calculs est déplorable. Il est donc important de pouvoir éliminer ces éléments; une solution radicale consiste à remailler entièrement le domaine d'étude à chaque pas de temps, mais elle ne peut convenir dans notre cas. Pour pouvoir calculer les vibrations électromagnétiques, il est particulièrement intéressant de conserver d'un pas de temps à l'autre les mêmes nœuds, c'est-à-dire le même maillage, tout du moins dans les parties faisant l'objet d'un calcul. Certains auteurs remail-
MAGNETISME

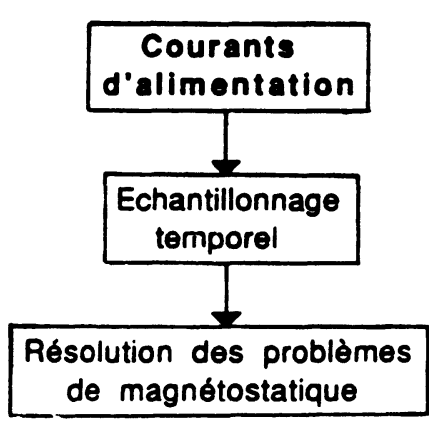

MECANIOUE

Décomposition en série de Fourier MECANIQUE

Densité électromagnétique de force
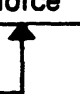

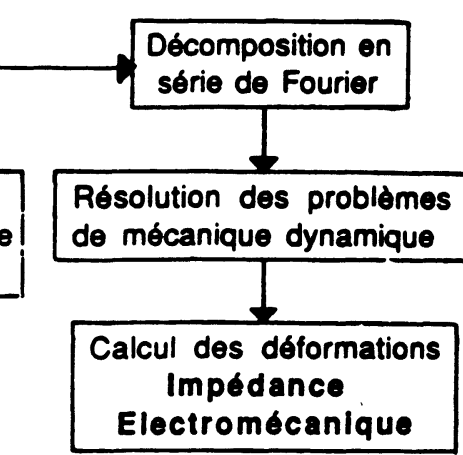

Fig. 13. - Principe de calcul de la fonction de transfert.

[Principle of the transfer function calculation.] 


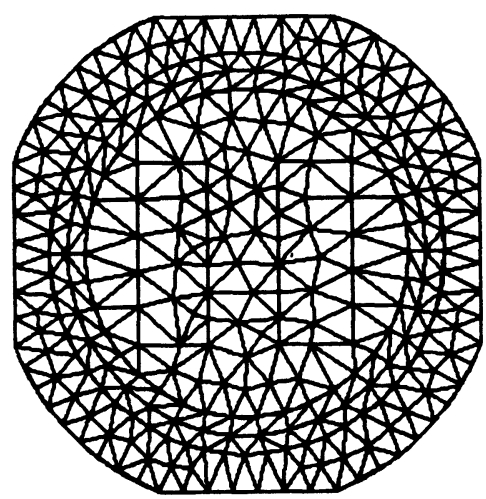

a)

Fig. 14. - (a) Maillage initial. (b) Maillage après rotation.

[(a) Initial mesh. (b) Mesh after rotation.]

lent uniquement l'entrefer en utilisant un algorithme d'optimisation; cette technique ne permet pas d'exploiter facilement les grandeurs magnétiques dans l'entrefer et surtout elle est difficile à mettre en œuvre dans un logiciel tel que FLUXMECA. Nous nous sommes tournés vers d'autres solutions plus connues qui ne nécessitent pas d'intervention au niveau du maillage.

Choix de la technique. - Actuellement, il existe essentiellement trois méthodes basées sur des techniques différentes :

- le macro-élément entrefer,

- la bande de roulement,

- la méthode des intégrales de frontière.

Les deux premières peuvent être utilisées dans un logiciel classique d'éléments finis, au prix de quelques modifications; quant à la troisième, elle exige des développements spécifiques assez lourds qui, malgré tout ont été réalisés dans FLUXMECA.

Le macro-élément entrefer [5], basé sur l'existence d'une solution analytique de l'équation de Laplace, est une couronne ne contenant que de l'air. Cette dernière, ne nécessitant pas de maillage dans son intérieur, permet d'isoler le rotor, partie tournante, du reste de la machine. Cette méthode a l'inconvénient de coupler entre eux l'ensemble des nœuds situés sur les deux cercles de la couronne d'une part et de recourir au calcul de séries doubles de Fourier d'autre part; les temps de résolution sont donc relativement longs, étant donné que, en plus des calculs de séries, la matrice du système linéaire à résoudre n'a pas une structure bande. Bien que l'un de ses avantages soit de ne pas imposer une discrétisation régulière sur les cercles, le macro-élément entrefer reste très peu utilisé à l'heure actuelle.

La bande de roulement, comme son nom l'indique, est une bande plus ou moins étroite à l'image du macro-élément entrefer ; mais contrairement à

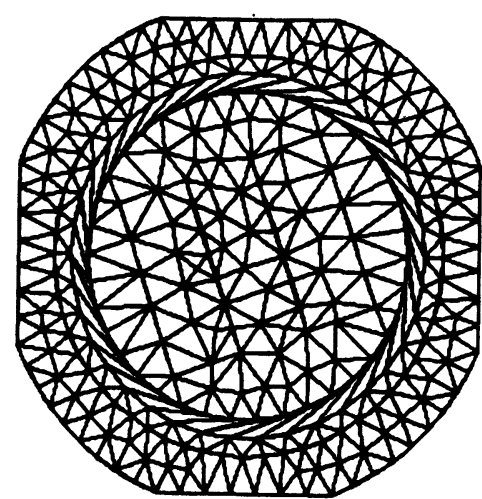

b)

celui-ci, elle nécessite d'être maillée dans son intérieur et elle exige une discrétisation parfaitement régulière, choisie en fonction du pas de temps; en effet, lorsque le rotor ou les parties mobiles tournent d'un pas de temps, il est nécessaire que les nœuds sur le cercle coïncident à nouveau entre eux, comme le montrent les figures $15 \mathrm{a}$ et $15 \mathrm{~b}$, afin d'éviter des interpolations compliquées.

Cette technique permet de conserver le même maillage dans l'entrefer à condition de mettre à jour un certain nombre de données. L'avantage de cette méthode est de conduire, au niveau de la résolution pour chaque pas de temps, à une matrice bande symétrique tout à fait classique, d'où des temps de résolution beaucoup plus compétitifs que dans le cas du macro-élément entrefer. De plus, une exploitation des grandeurs magnétiques dans l'entrefer est tout à fait possible, sans traitement particulier. Précisons que la bande de roulement n'est pas nécessairement une couronne d'épaisseur constante.

La méthode des intégrales de frontière, couplée avec la méthode classique des éléments finis [6] permet aussi d'envisager l'étude de dispositifs comportant des parties mobiles. De même que dans les deux cas précédents, il faut isoler les parties mobiles des parties fixes à l'aide d'une bande contenant de l'air, mais dont la forme peut être quelconque, à condition toutefois que le contour intérieur puisse tourner librement à l'interieur $u$ contour extérieur. La discrétisation des contours n'exige aucune contrainte particulière, ce qui fait que les pas de temps peuvent ne pas être constants, comme c'est souvent le cas avec une méthode classique de pas à pas dans le temps avec prédictioncorrection. Cette méthode, dite hybride, s'avère particulièrement générale, d'où son attrait pour l'étude dynamique des machines tournantes avec rotor en mouvement. De nombreux auteurs l'ont adoptée, en particulier pour l'étude des moteurs asynchrones. 


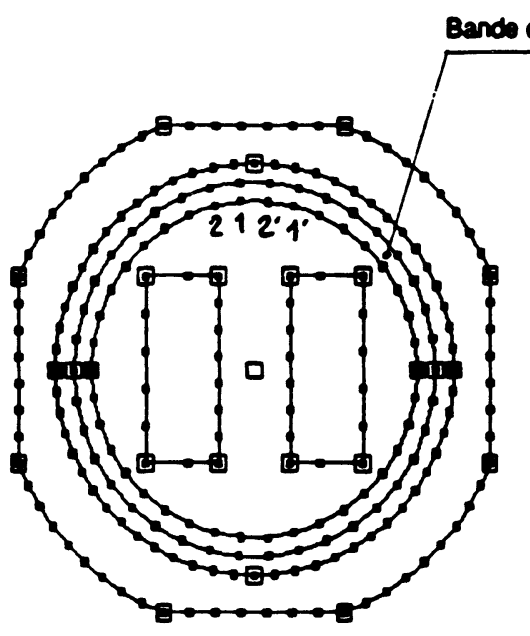

(1)

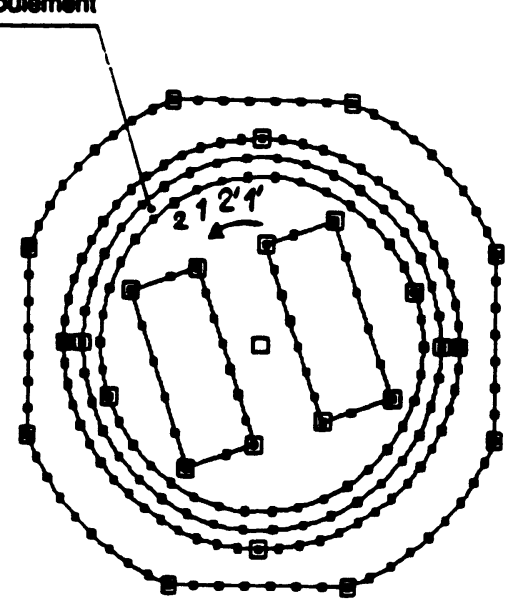

h)

Fig. 15. -- (a) Position initiale des nœuds. (b) Position après rotation.

[(a) Initial position of nodes. (b) Position after rotation.]

Parmi ces trois méthodes, nous avons retenu les deux dernières. Mais le couplage éléments finis/intégrales de frontière, tout comme le macro-élément, conduit à la résolution d'un système linéaire dont la matrice est pleine et, en plus, non symétrique. Cet inconvénient s'est révélé être un véritable handicap sitôt que le problème à résoudre comporte un nombre important de nœuds; étant donné que les machines tournantes constituent en général de gros problèmes, nous avons décidé de développer une autre méthode dérivée de la bande de roulement, plus simple encore à mettre en œuvre dans un logiciel de calcul par éléments finis sur le plan informatique (pas de mise à jour des numéros de nœuds, ni des voisins pour chaque pas de temps) et surtout dans le but d'obtenir les résultats beaucoup plus rapidement. Cette méthode que nous avons appellée méthode de la " bande de roulement modifiée ", consiste à définir non pas une bande de roulement, mais deux tout à fait identiques.
L'une des bandes de roulement est située dans l'entrefer de la machine et l'autre est entièrement à l'extérieur comme le montre la figure 16 ; seule cette dernière nécessite d'être maillée; elle joue le rôle de la bande de roulement classique. Les deux bandes de roulement, identiques d'un point de vue géométrique, doivent également l'être au niveau de la discrétisation ; cela permet de les relier entre elles, au niveau de la résolution éléments finis, par des conditions aux limites simples dites d'égalité, portant sur la variable d'état, tout en évitant, là encore, des interpolations compliquées.

EXEMPLES COMPLETS D'APPLICATION. - Après avoir utilisé divers exemples pour illustrer certains points ou mettre en évidence les potentialités d'application du logiciel FLUXMECA, deux exemples traités de manière plus complète permettent de faire ressortir les diverses étapes du calcul, ainsi que les résultats obtenus. Le premier est un cas simple, de
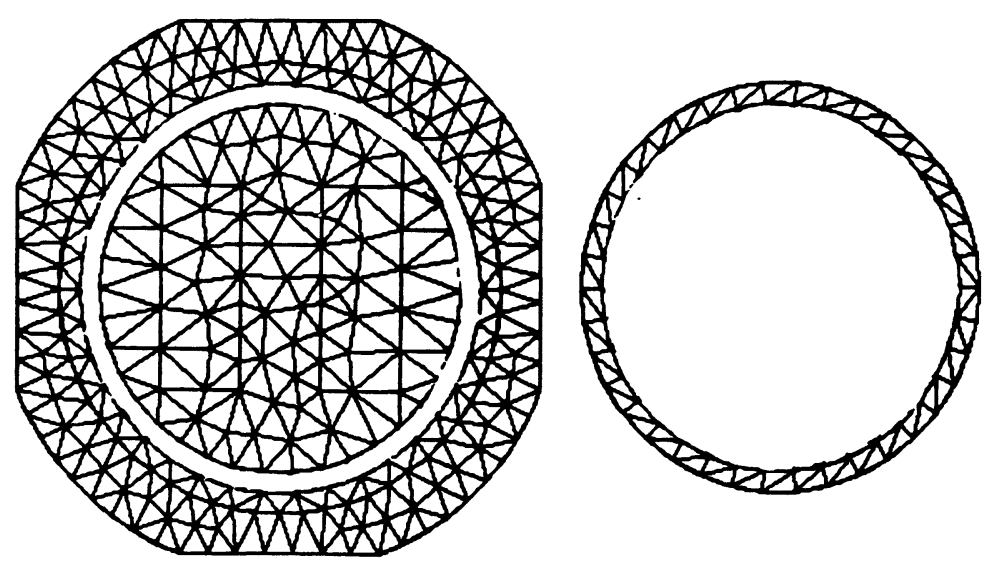

Fig. 16. - Technique de la bande de roulement modifiée.

[Technique of the modified slide band.] 
nature didactique, afin de bien préciser les différentes étapes sans que la présentation n'en soit trop alourdie. Le second traite un cas nettement plus complexe, mais bien réel.

Premier exemple: structure simple en rotation. - Il s'agit d'une structure simplifiée mise en rotation à vitesse constante autour de son centre de symétrie : une inductance alimentée en courant continu. La rotation et la décomposition spectrale avec réponse mécanique sont utilisées pour obtenir la réponse vibratoire désirée. A titre indicatif, le maillage utilisé pour la résolution éléments finis apparaît sur la figure 16. Les figures $17 \mathrm{a}$ et $17 \mathrm{~b}$ montrent les lignes de champ correspondant à deux pas de temps différents; les figures $18 \mathrm{a}$ et $18 \mathrm{~b}$ représentent la densité surfacique de force aux mêmes instants.

Le logiciel FLUXMECA, grâce à une analyse spectrale, permet de reconstituer l'évolution dans le temps des grandeurs magnétiques ou mécaniques à partir des différents échantillons. Par exemple, la figure 19a représente l'évolution de la densité surfacique de force au point $A$, repéré sur la figure $17 \mathrm{a}$; la figure $19 \mathrm{~b}$ montre le spectre en fréquence correspondant et met en évidence que la densité de force est la superposition de deux composantes, l'un'e continue et l'autre sinusoïdale.

Nous pouvons également étudier la densité de force au point $\mathrm{A}^{\prime}$, comme le montrent les figures $20 \mathrm{a}$ et $20 \mathrm{~b}$.

Ainsi, les figures $19 \mathrm{a}$ et $20 \mathrm{a}$ confirment très clairement que les densités de force en des points différents, $A$ et $A^{\prime}$ par exemple, de la structure sont déphasées dans le temps comme nous l'avons déjà fait remarquer. Il est donc impératif pour calculer les vibrations induites par le mouvement de parties mobiles d'utiliser la mécanique basée sur les grandeurs complexes qui permet pour chaque harmoni-

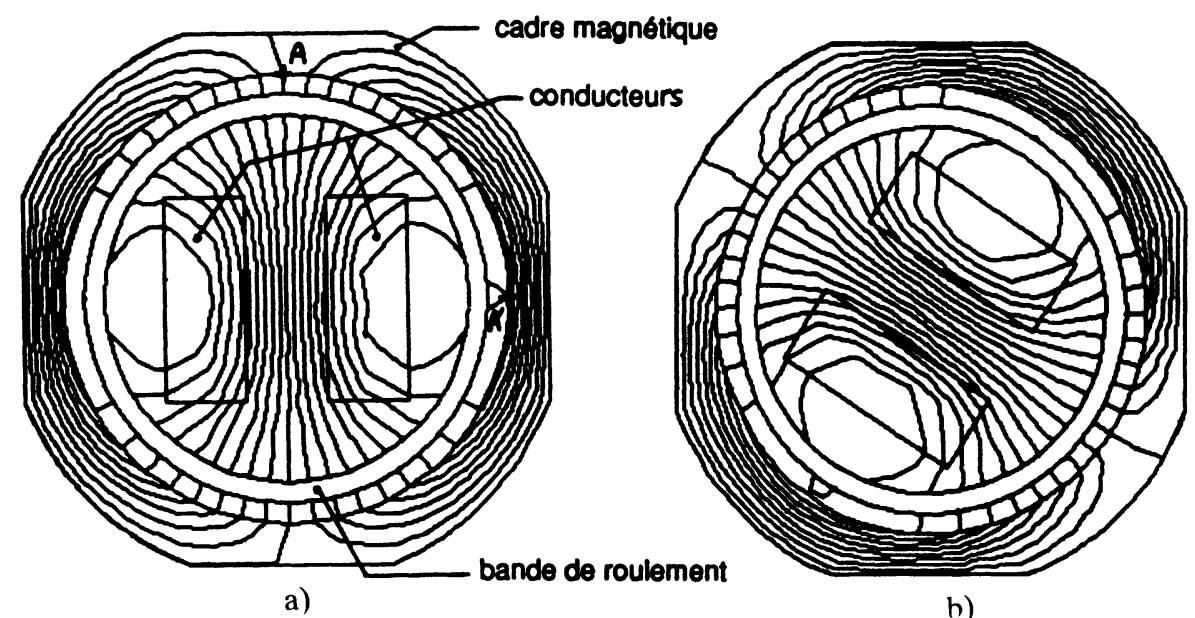

a)

Fig. 17. - (a) Lignes de champ à $t=0$. (b) Lignes de champ à $t=\Delta t$.

[(a) Magnetic field pattern at $t=0$. (b) Magnetic field pattern at $t=\Delta t$.]

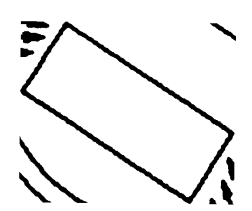

a)

h)

Fig. 18. (a) Répartition de la densité de force à $t=0$. (b) Répartition de la densité de force à $t=\Delta t$.

[(a) Distribution of the force density at $t=0$. (b) Distribution of the force density at $t=\Delta t$.] 


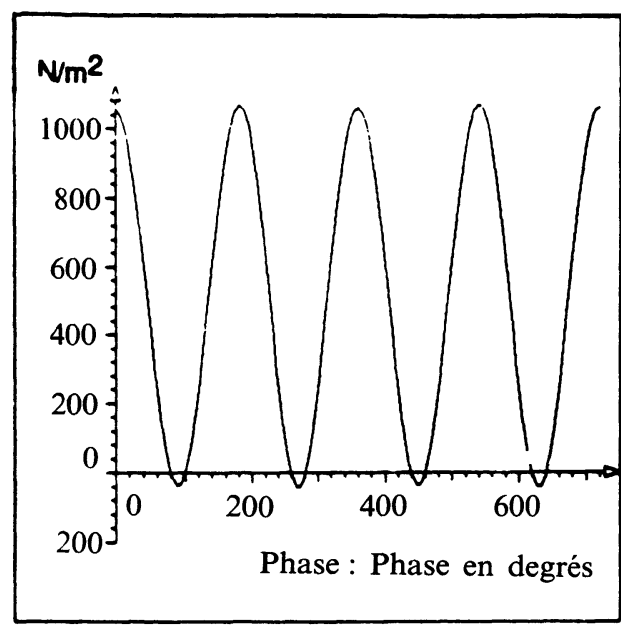

a)

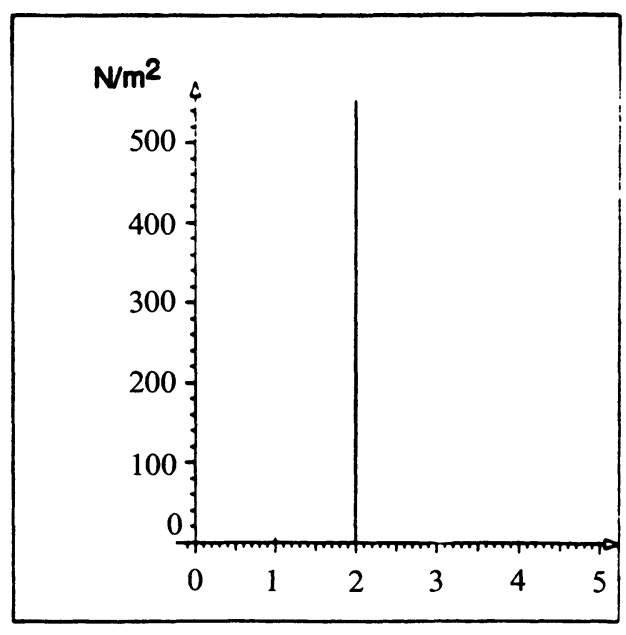

h)

Fig. 19. - (a) Densité de force au point A. (b) Spectre en fréquence.

[(a) Force density at point A. (b) Frequency spectrum.]

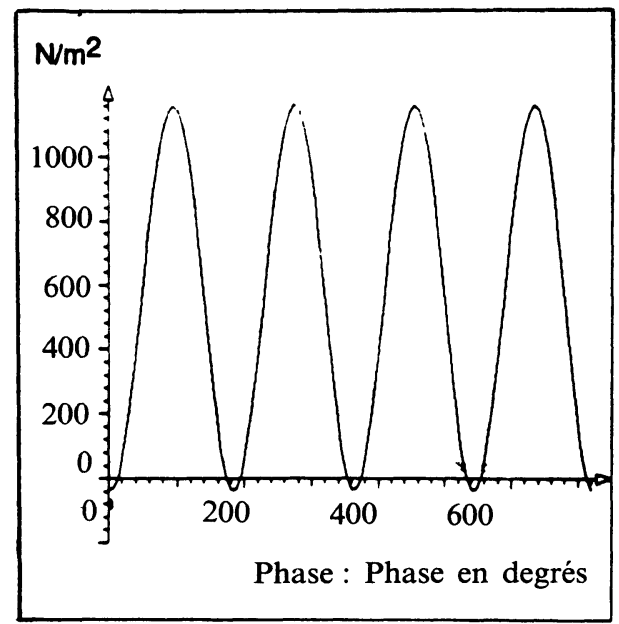

a)

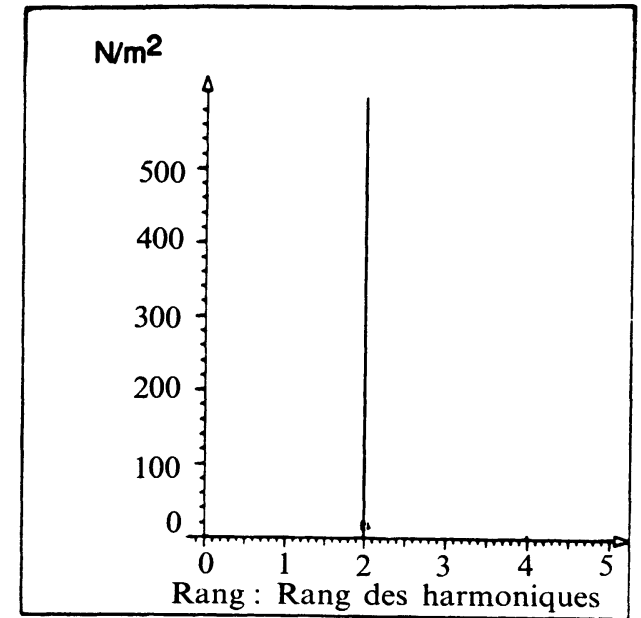

h)

Fig. 20. - (a) Densité de force au point $\mathrm{A}^{\prime}$. (b) Spectre en fréquence.

[(a) Force density at point $A^{\prime}$. (b) Frequency spectrum.]

que de force de prendre en compte un éventuel déphasage. Nous avons donc été amenés à développer dans FLUXMECA un module d'intégration spécifique et nous avons ensuite adapté nos outils d'exploitation (analyse spectrale) à l'étude des grandeurs complexes. A ce stade de nos travaux, nous sommes dorénavant en mesure de calculer les vibrations d'origine magnétique correspondantes.

A titre d'exemple, les figures $22 \mathrm{a}, 22 \mathrm{~b}$ et $22 \mathrm{c}$ représentent les accélérations aux points $\mathrm{C}$ et $C^{\prime}$ et $C^{\prime \prime}$ situés à la périphérie du cadre magnétique, là où il pourrait être envisagé, sur le plan expérimental, de disposer des accéléromètres; ces points sont repérés sur la figure 21 qui montre également que le cadre magnétique est considéré dans cette application comme encastré à sa base.

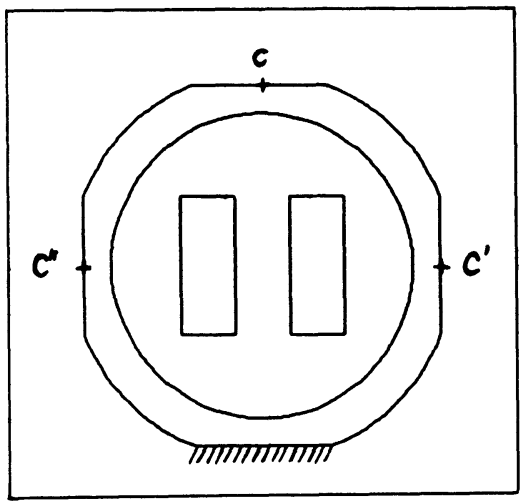

Fig. 21. - Points $\mathrm{C}, \mathrm{C}^{\prime}$ et $\mathrm{C}^{\prime \prime}$ sur le cadre magnétique. [Points $\mathrm{C}, \mathrm{C}^{\prime}$ and $\mathrm{C}^{\prime \prime}$ on the magnetic frame.] 


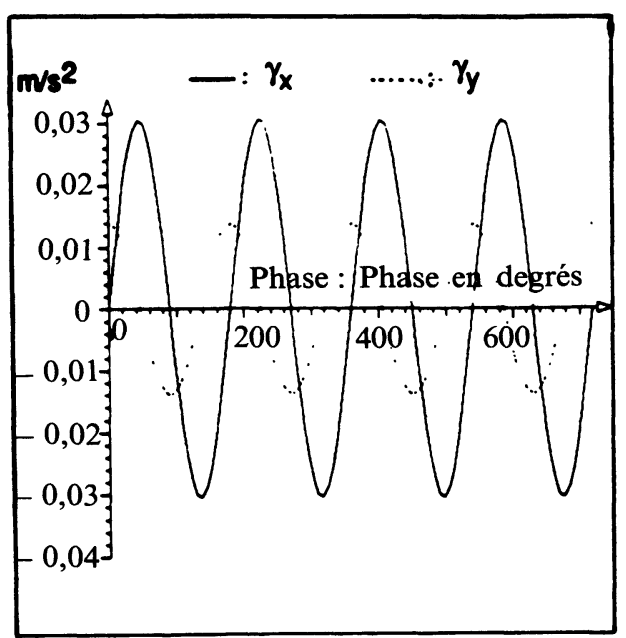

a)

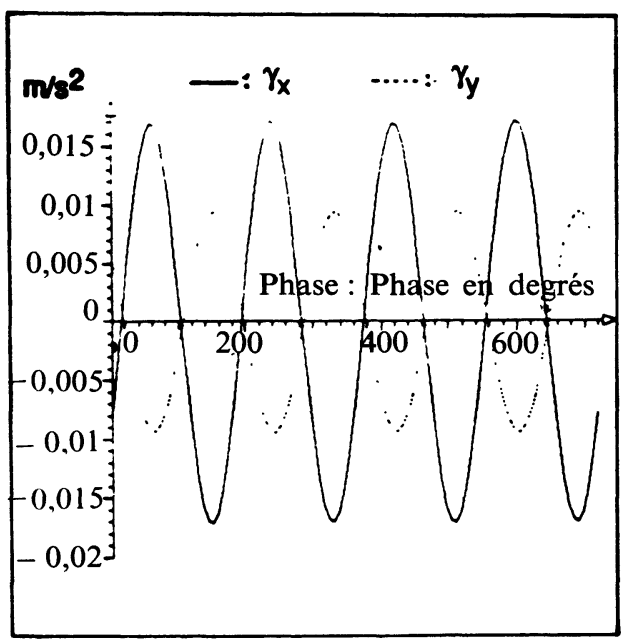

h)

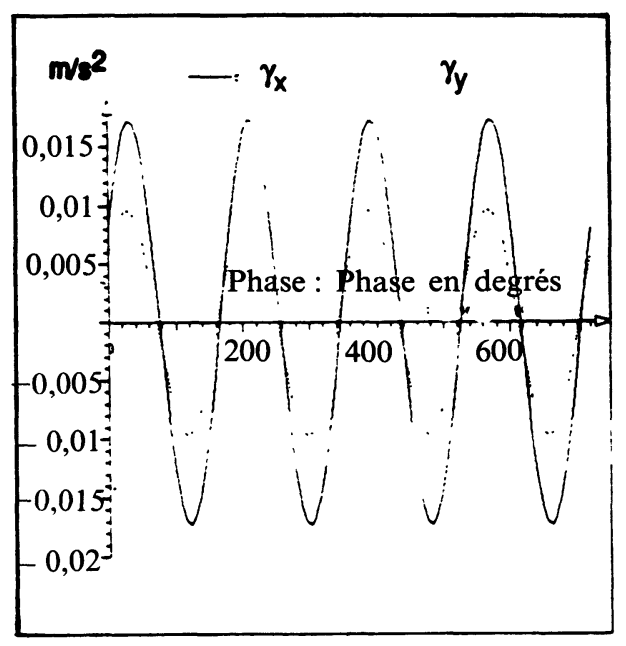

c)

Fig. 22. - (a) Accélérations au point $\mathrm{C}$ suivant les deux axes $x$ et $y$. (b) Accélérations au point $\mathrm{C}^{\prime}$. (c) Accélérations au point C".

[(a) Accelerations at point $\mathrm{C}$ in the $x$ - and $y$-directions. (b) Accelerations at point $\mathrm{C}^{\prime}$. (c) Accelerations at point C".]

La figure $22 \mathrm{a}$ montre que les accélérations suivant $x$ et $y$ sont en quadrature : ce résultat est tout à fait conforme à celui auquel nous pouvons nous attendre par des considérations élémentaires qui ne prennent en compte ni la réponse de la structure, ni les conditions aux limites ; en effet, la densité de force à la surface du cadre est assimilable à une onde qui se dé lace à vitesse constante et ui, entraînant dans son sillage les déformations du cadre, tend à lui donner une forme ovale.

Par contre, les figures $22 \mathrm{~b}$ et $22 \mathrm{c}$ montrent clairement que les conditions aux limites influent sur la réponse de la structure : les accélérations suivant $x$ et $y$ sont quasiment en phase ou en opposition de phase et non plus en quadrature ; ce phénomène est difficile à prévoir a priori : cela nous permet d'insister sur le fait qu'il est indispensable de prendre en compte la réponse propre de la structure et bien évidemment les conditions aux limites.
A titre de comparaison, nous avons effectué un calcul de vibrations en considérant que les forces sont en phase en tout point de la structure : les amplitudes des harmoniques sont calculées correctement; seuls les déphasages ne sont pas pris en compte. Comme nous nous y attendions, nous avons obtenu des résultats assez différents des précédents du point de vue quantitatif. Ainsi, pour espérer effectuer un calcul correct e vi rations, 1 est nécessaire de tenir compte du déphasage des forces dans le temps. Notons que ce déphasage, dans l'exemple traité ci-dessus, correspond en fait à un déphasage spatial, du fait que les courants induits sont négligés.

Le logiciel FLUXMECA étant dorénavant opérationnel pour l'étude des vibrations des systèmes électromagnétiques comportant ou non des parties mobiles, nous pouvons envisager le calcul de la fonction de transfert électromécanique d'une machine à courant continu. 
Deuxième exemple : moteur à courant continu. - Le logiciel FLUXMECA que nous avons adapté à nos besoins en matière de vibrations, tout au long de nos travaux, est un outil informatique qui permet d'analyser les phénomènes vibratoires d'origine électromagnétique dans les machines tournantes, pourvu que les courants induits soient négligeables. Nous proposons de l'appliquer aux machines à courant continu et, en particulier, au moteur industriel présenté en figure $9 \mathrm{a}$ ou $9 \mathrm{~b}$ qui est installé sur un banc d'essai dans notre laboratoire. Mais avant d'aborder l'étude dynamique avec le rotor en mouvement, nous considérons la machine avec son rotor à l'arrêt afin d'éliminer toute source de vibrations d'origine mécanique; le spectre de vibrations qui ne comporte alors que des raies d'origine électromagnétique est plus simple à interpréter et, surtout, il devrait nous permettre de valider plus facilement les calculs de vibrations. Ensuite, nous présentons une approche de la raie d'encoche en régime dynamique.

- Réponse d'un moteur à courant continu à rotor bloqué. - Le moteur est à l'arrêt et son induit n'est pas alimenté. En revanche, les inducteurs sont parcourus par un courant continu de 1 ampère dans le but de masquer les champs rémanents au sein de la machine, pour créer des perturbations ou vibrations magnétiques, nos injectons dans des enroulements supplémentaires situés autour des bobinages des pôles principaux, un courant alternatif de 1,5 ampère crête de $600 \mathrm{~Hz}$; ils sont accessibles à l'extérieur de la machine par le biais d'une plaque à bornes. Nous avons utilisé deux de ces enroulements comme le montre la figure 23 et nous les avons connectés de manière à ce que les flux alternatifs créés $\phi_{1}$ et $\phi_{2}$ s'ajoutent afin d'augmenter volontairement l'effet des vibrations pour faciliter les mesures. Notons que le moteur est suspendu de part et d'autre par des plots élastiques, modélisés par deux éléments élastiques suppósés encastrés à leur base.

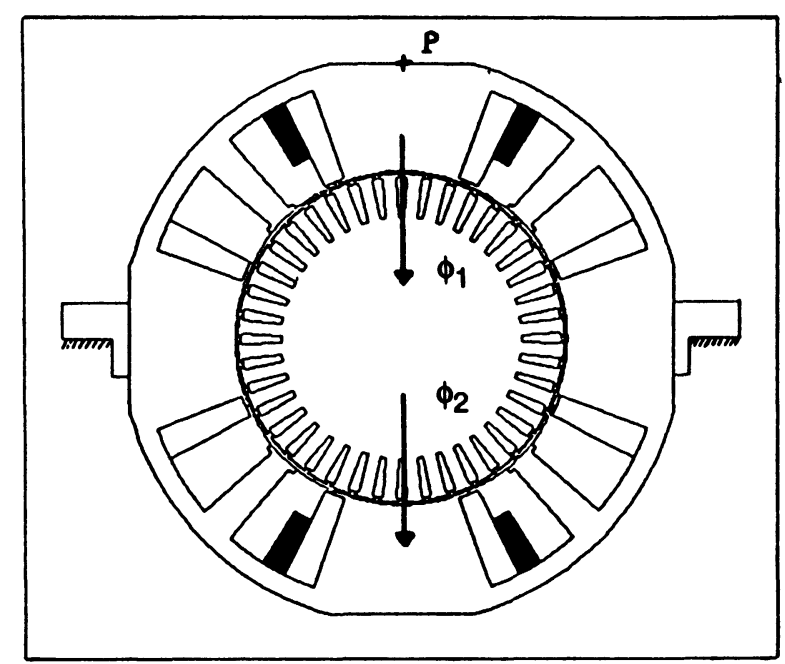

Fig. 23. - Moteur industriel et les enroulements supplémentaires.

[Industrial motor and the supplementary windings.]

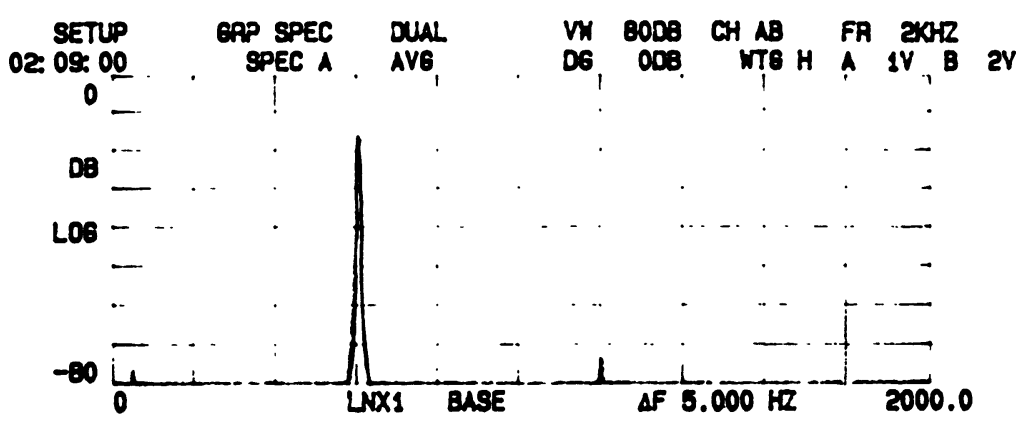

a)

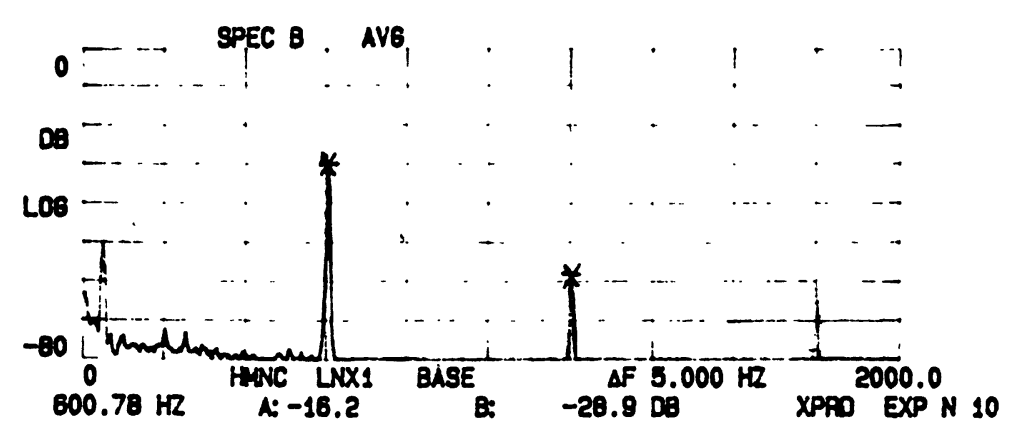

h)

Fig. 24. - (a) Spectre du courant expérimental. (b) Spectre vibratoire expérimental.

[(a) Spectrum of experimental current. (b) Experimental spectrum of vibrations.] 
La figure 24a montre le spectre du courant relevé expérimentalement; nous pouvons considérer qu'il est sinusoïdal. La figure $24 \mathrm{~b}$ correspond au spectre vibratoire expérimental (accélération suivant l'axe vertical) de la machine au point $P$, repéré sur la figure 23 ; dans ce cas, précisons que le niveau $0 \mathrm{~dB}$ équivaut à une accélération de $0,2 \mathrm{~g}\left(g=9,81 \mathrm{~m} / \mathrm{s}^{2}\right)$.

$\mathrm{Au}$ niveau de la modélisation numérique, nous avons fait les calculs en utilisant les valeurs suivantes pour les paramètres mécaniques, à savoir module d'Young, coefficient de Poisson et masse volumique :

- matériau ferromagnétique :

$$
\begin{gathered}
E=2,2 \times 10^{11} \mathrm{~N} / \mathrm{m}^{2}, \\
\nu=0,35 \text { et } \rho=8000 \mathrm{~kg} / \mathrm{m}^{3} .
\end{gathered}
$$

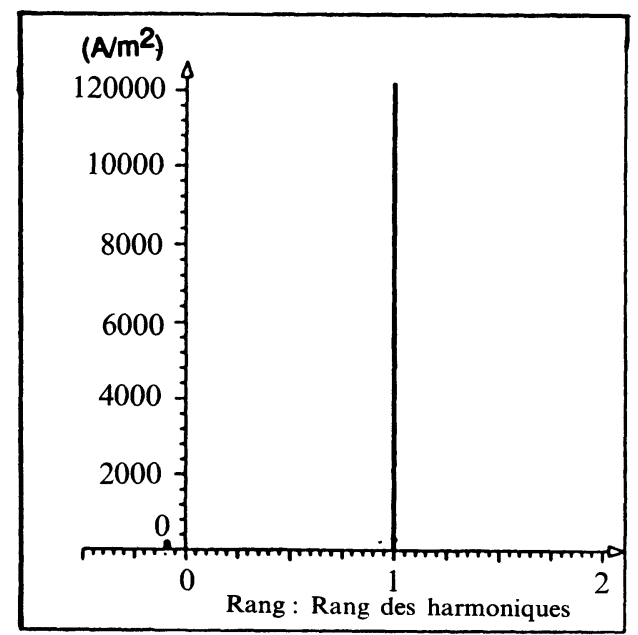

a)
- conducteurs :

$$
\begin{gathered}
E=1,2 \times 10^{11} \mathrm{~N} / \mathrm{m}^{2}, \\
\nu=0,36 \text { et } \rho=8900 \mathrm{~kg} / \mathrm{m}^{3} .
\end{gathered}
$$

- matériau élastique :

$$
\begin{gathered}
E=0,8 \times 10^{11} \mathrm{~N} / \mathrm{m}^{2}, \\
\nu=0,45 \text { et } \rho=2000 \mathrm{~kg} / \mathrm{m}^{3} .
\end{gathered}
$$

La figure 25a montre le spectre de la densité de courant qui parcourt les enroulements supplémentaires et que nous avons utilisé pour la modélisation. La figure $25 \mathrm{~b}$ correspond au spectre des accélérations au point $\mathbf{P}$ suivant les deux axes $x$ et $y$.

Le niveau vibratoire de la composante fondamentale suivant l'axe vertical (ici, la plus importante)

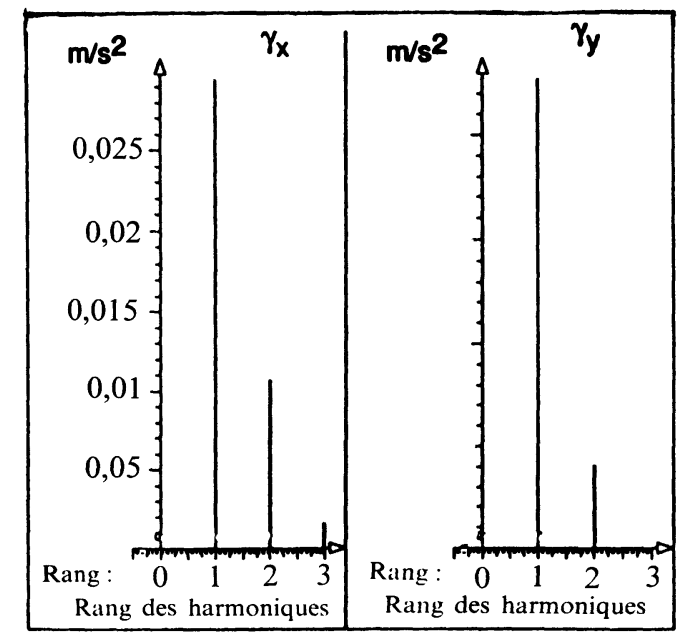

b)

Fig. 25. - Spectre de la densité de courant utilisée dans le modèle.

[Spectrum of the current density for the modeling.]

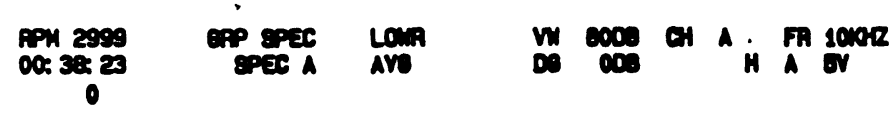

\section{Raie dencoche}

108

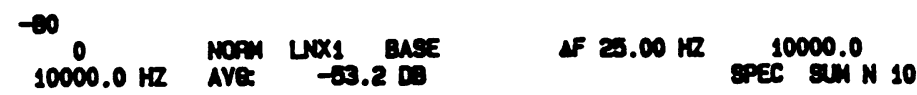

Fig. 26. - Spectre d'accélération et la fréquence d'encoche.

[Vibration spectrum with the slot frequency.] 


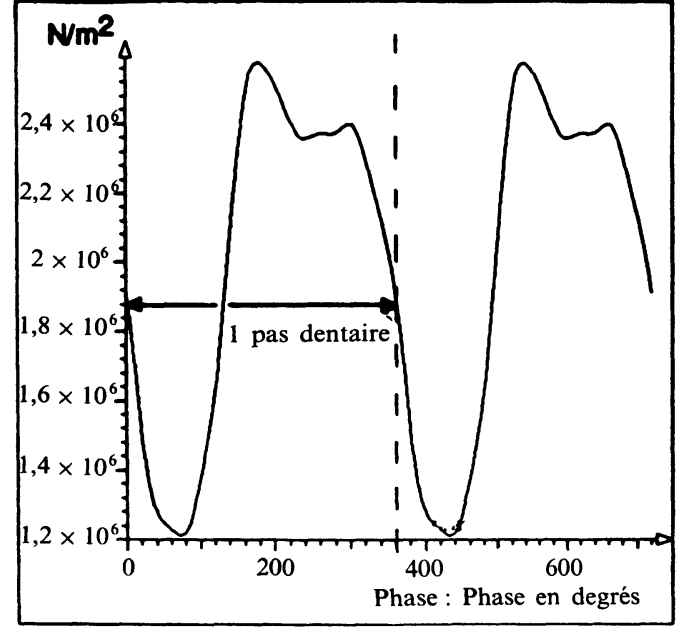

a)

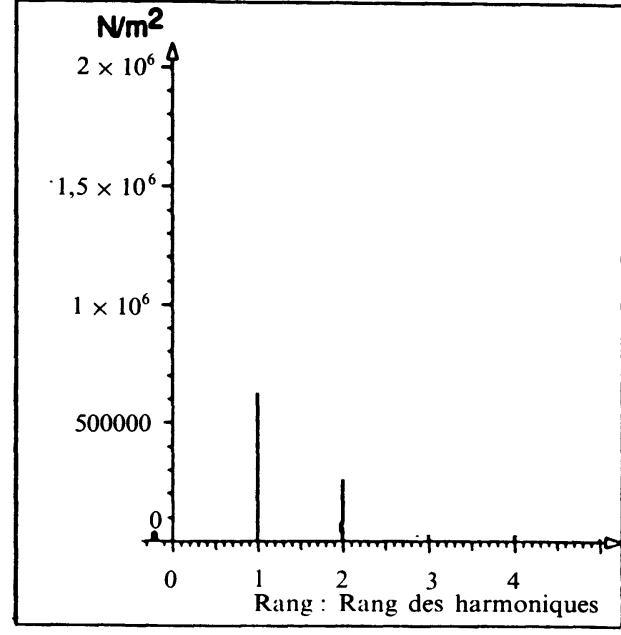

h)

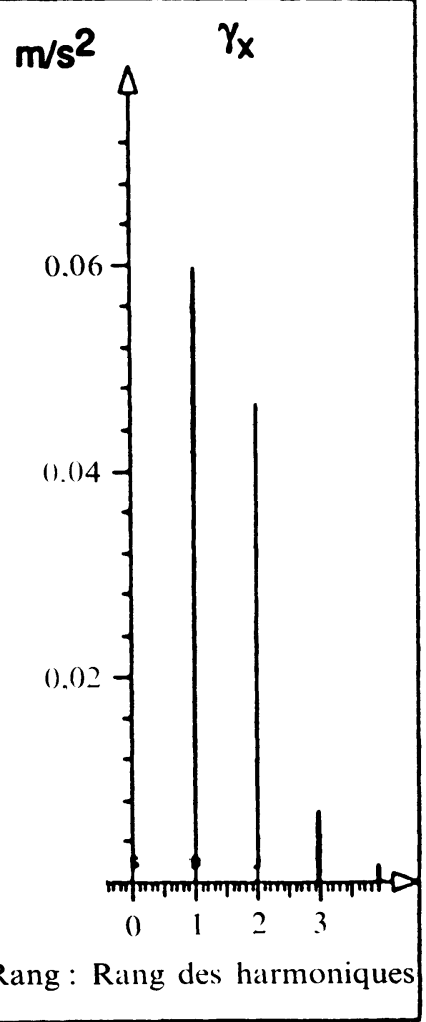

Rang: Rang des harmoniques

Rang: Rang des harmoniques

c)

Fig. 27. - (a) Evolution de la densité de force dans le temps. (b) Harmoniques de la densité de force. (c) Spectre de vibrations calculé.

[(a) Time dependancy of force density. (b) Harmonic components of the force density. (c) Calculated spectrum of vibrations.]

obtenu par la modélisation est de $0,090 \mathrm{~m} / \mathrm{s}^{2}$; compte tenu de la valeur expérimentale qui est de $0,063 \mathrm{~m} / \mathrm{s}^{2}$ et de la difficulté d'appréhender certaines grandeurs magnétiques ou mécaniques, l'ordre de grandeur est tout à fait correct. En revanche, il est à noter une différence plus importante concernant l'harmonique de rang 2 ; il serait utile d'envisager une étude de sensibilité des paramètres mécaniques.
Mais globalement, les résultats obtenus par la modélisation sont satisfaisants. Cela nous a donc encouragé à aller plus loin et à étudier les vibrations magnétiques qui sont dues à la rotation de la machine.

- Réponse dynamique du moteur à courant continu. - La réponse dynamique du moteur avec rotor en mouvement est intéressante puisqu'elle 
débouche directement sur l'étude de la raie d'encoche; cette dernière est à l'origine de vibrations importantes, particulièrement difficiles à atténuer, que bon nombre de constructeurs aimeraient maîtriser. Rappelons que le moteur considéré comportè quarante encoches au rotor et précisons que nous avons mené l'étude en régime de fonctionnement nominal correspondant à une vitesse de rotation de $3000 \mathrm{tr} / \mathrm{min}$ ou encore à une fréquence de $50 \mathrm{~Hz}$. Sur le spectre vibratoire, la raie d'encoche qui apparaît à une fréquence égale au produit du nombre d'encoches par la fréquence de rotation est donc visible à la fréquence de $2 \mathrm{kHz}$; le relevé expérimental de la figure 26 met parfaitement en évidence la raie d'encoche, ainsi que quelques-uns de ses harmoniques. Le niveau vibratoire de la raie d'encoche correspond à une accélération de $0,07 \mathrm{~m} / \mathrm{s} 2$.

Sur le plan de la modélisation, nous pouvons analyser les phénomènes vibratoires sur une période magnétique, c'est-à-dire sur un pas dentaire compte tenu de la structure régulière du rotor. Cela nous permet de limiter le nombre d'échantillons temporels nécessaires pour effectuer des calculs corrects; à titre indicatif, nous avons utilisé seize échantillons, ce qui s'est avéré tout à fait suffisant par la suite. Par ailleurs, signalons que, pour obtenir une bonne précision sur les calculs à chaque pas de temps, il a été nécessaire d'utiliser un maillage très fin au niveau de l'entrefer du moteur; l'analyse vibratoire d'une structure complexe telle que celle d'un moteur conduit inévitablement à de très gros problèmes du point de vue du nombre d'inconnus, problèmes qui sont assez lourds à manipuler.

La figure 27 a représente l'évolution temporelle de la densité surfacique de force en un point situé sur le pôle ; la figure $27 \mathrm{~b}$, quant à elle, montre la décomposition spectrale associée. Le calcul de la réponse mécanique, fréquence par fréquence, conduit au spectre vibratoire de la figure $27 \mathrm{c}$ qui correspond à l'accélération verticale au point $P$; le niveau vibratoire est de $0,15 \mathrm{~m} / \mathrm{s}^{2}$, soit le double de celui obtenu expérimentalement. Les différences à propos des autres raies sont plus importantes.

Cependant, d'une manière générale, il est à noter que, en l'état actuel des choses, la modélisation bidimensionnelle que nous avons développée, à travers le logiciel FLUXMECA, pour l'étude des vibrations magnétiques permet d'obtenir des ordres de grandeur tout à fait satisaisants même s'ils ne sont pas encore très précis ; mais bien entendu, il est loin d'être évident de rendre compte de phénomènes $3 \mathrm{D}$ par une analyse $2 \mathrm{D}$, surtout en mécanique des structures.

\section{Conclusion.}

Par nos travaux sur la modélisation des vibrations d'origine magnétique, nous avons cherché à démontrer que l'étude des phénomènes couplés, magnétiques et mécaniques, est possible malgré toute leur complexité, à condition d'utiliser un certain nombre d'hypothèses simplificatrices.

Tout d'abord, nous nous sommes intéressés à la répartition des forces qui sont à l'origine des vibrations d'un point de vue strictement qualitatif et, ensuite, nous avons développé le calcul de la fonction de transfert électromécanique qui permet d'étudier les vibrations dans les machines tournantes et d'obtenir des résultats quantitatifs. Les calculs conduisent à des ordres de grandeur corrects, mais les efforts entrepris jusqu'à maintenant doivent être poursuivis afin d'améliorer la corrélation entre les résultats expérimentaux et ceux obtenus par la modélisation.

L'objectif de nos travaux est de fournir très prochainement un véritable outil de CAO à tout concepteur de machines électriques désireux d'améliorer, voire d'optimiser, leur comportement vibratoire quelque soient les domaines d'application. L'analyse des vibrations en trois dimensions est déjà bien avancée dans notre laboratoire et les résultats promettent être intéressants, compte tenu de ceux obtenus par une analyse simplifiée bidimensionnelle. 


\section{Bibliogaphie}

[1] Reyne G., Analyse théorique et expérimentale des phénomènes vibratoires d'origine électromagnétique, Thèse de l'Institut National Polytechnique de Grenoble (décembre 1987).

[2] Lefevre Y., Etude de la répartition des efforts magnétiques dans les machines synchrones à aimants permanents et commutation électronique. Détermination des vibrations d'origine magnétique, Thèse de l'Institut National Polytechnique de Toulouse (juin 1988).

[3] IMHoff J. F., Modélisation magnétique et mécanique des machines électriques par la méthode des éléments finis, Thèse de l'Institut National Polytechnique de Grenoble (octobre 1989).

[4] Imhoff J. F., Reyne G., Meunier G., Foggia A. and SABONNADIERE J. C., Spectral analysis of electromagnetic vibrations in D.C. Machines throughout the finite element method, Laboratoire d'Electrotechnique de Grenoble, St-Martin-d'Hères, France. IEEE Trans. Magn. 19, $\mathrm{n}^{\circ} 6$ (janvier 1987).

[5] Feliachi M., Coulomb J. L., Mansir H., Second order air gap element for the dynamic finite element analysis of the electromagnetic field in electric machines, Laboratoire d'Electrotechnique de Grenoble, St-Martin-d'Hères, France. IEEE Trans. Magn. 19, $\mathrm{n}^{\circ} 6$ (novembre 1983).

[6] Meunier G., Coulomb J. L., Salon S. and KrahenBUL L., Hybrid finite element-boundary element solution for three dimensional scalar potential problems, IEEE Trans. Magn. 22, $\mathrm{n}^{\circ} 5$ (septembre 1986). 